\title{
Gradient Estimates for a Weighted $\Gamma$-nonlinear Parabolic Equation Coupled with a Super Perelman-Ricci Flow and Implications
}

\section{Ali Taheri ${ }^{1}[1$}

Received: 3 December 2020 / Accepted: 11 November 2021 / Published online: 25 November 2021

(C) The Author(s) 2021

\begin{abstract}
This article studies a nonlinear parabolic equation on a complete weighted manifold where the metric and potential evolve under a super Perelman-Ricci flow. It derives elliptic gradient estimates of local and global types for the positive solutions and exploits some of their implications notably to a general Liouville type theorem, parabolic Harnack inequalities and classes of Hamilton type dimension-free gradient estimates. Some examples and special cases are discussed for illustration.
\end{abstract}

Keywords Weighted Riemannian manifold · Gradient estimates · Super Perelman-Ricci flow $\cdot$ Bakry-Émery tensor $\cdot$ Harnack inequality $\cdot$ Liouville theorem

Mathematics Subject Classification (2010) 53C44 · 58J60 · 58J35 $\cdot 60 \mathrm{~J} 60$

\section{Introduction}

Let $(M, g)$ be a complete Riemannian manifold of dimension $n \geq 2$ endowed with a weighted measure $d \mu=e^{-f} d v_{g}$ where $f$ is a potential of class $\mathscr{C}^{\infty}$ on $M$ and $d v_{g}$ is the Riemannain volume measure. In this paper we consider the following nonlinear parabolic equation where the metric-potential pair are time dependent and evolve under a $(k, m)$ super Perelman-Ricci flow,

$$
\left\{\begin{array}{l}
\square_{q} u(x, t):=\left[\frac{\partial}{\partial t}-q(x, t)-\Delta_{f}\right] u(x, t)=\Gamma(u)(x, t), \quad t>0, \\
\frac{\partial g}{\partial t}(x, t)+2 \mathscr{R} i c_{f}^{m}(g)(x, t) \geq-2 k g(x, t) .
\end{array}\right.
$$

Here $\Delta_{f}$ is the so-called weighted Laplacian acting on smooth functions $v \in \mathscr{C}^{\infty}(M)$ by $\Delta_{f} v=e^{f} \operatorname{div}\left(e^{-f} \nabla v\right)=\Delta v-\langle\nabla f, \nabla v\rangle$, where $\Delta$, div and $\nabla$ denote the usual

Ali Taheri

A.Taheri@sussex.ac.uk

1 School of Mathematical and Physical Sciences University of Sussex, Falmer, Brighton, UK 
Laplace-Beltrami, divergence and gradient operators with respect to the metric $g$ respectively. Also $\square_{q}=\partial_{t}-q-\Delta_{f}$ is the $q$-weighted heat operator where $q=q(x, t)$ is a smooth function, at least of class $\mathscr{C}^{1}$ in $x$ and $\mathscr{C}^{0}$ in $t$. Throughout $\Gamma=\Gamma(u)$ is a sufficiently smooth function, called the nonlinearity (see below for more), and for the sake of future reference we write $\mathscr{L}_{\Gamma}$ for the operator,

$$
\mathscr{L}_{\Gamma}(v)=\Delta_{f} v+\Gamma(v)=\Delta v-\langle\nabla f, \nabla v\rangle+\Gamma(v) .
$$

Now with the aid of Eq. 1.2 the first equation in Eq. 1.1 can be rewritten as

$$
\frac{\partial u}{\partial t}-q(x, t) u=\mathscr{L}_{\Gamma}(u), \quad t>0 .
$$

Note that in the second equation in Eq. 1.1, $\mathscr{R} i c_{f}^{m}$ denotes the generalised Bakry-Émery $m$ Ricci curvature tensor [cf. Eq. 1.7 below] with $m \geq n$ (in fact $m=\infty$ is allowed in which case we often write $\mathscr{R} i c_{f}^{\infty}=\mathscr{R} i c_{f}$ ) and $k$ is a fixed real number.

Our first objective is to develop local and global gradient estimates of elliptic type for positive smooth solutions to Eq. 1.1. As primarily here we are concerned with positive solutions $u$, the nonlinearity $\Gamma=\Gamma(u)$ is taken a smooth function, at least of class $\mathscr{C}^{1}$, defined on the half-axis $u>0$. To showcase our results and to put the problems studied into context, we of course discuss various classes of nonlinearities $\Gamma$ with specific growth and singularities at infinity or zero, e.g., logarithimic-power type singularities or polynomial power-like growth or a combination of the two, as arising in many contexts and recent applications (see, e.g., [7, 13, 20, 21, 37, 39, 40] and the references therein). However, for the sake of the gradient estimates per se and many of the consequences, we keep the discussion general and do not make any specific structural assumptions on the nonlinearity $\Gamma$.

Our next objective is to present some consequences of these estimates notably here to parabolic Harnack inequalities and some relative evolutionary estimates that include a Hamilton-Zhang type global gradient estimate to the nonlinear context with dimension free constants along with some novel $p, q$ variations of that theme. As the static case is a particular instance of the system we also discuss some consequences of the gradient estimate to that context. In particular here we establish Liouville type theorems for the corresponding elliptic equation $\mathscr{L}_{\Gamma}[u]=\Delta_{f} u+\Gamma(u)=0$ subject to the non-negativity of the BakryÉmery curvature tensors $\mathscr{R} i c_{f}^{m} \geq 0$ and $\mathscr{R} i c_{f} \geq 0$ (see below and Section 4 for more). To the best of our knowledge these results are the first in the literature under such general assumptions on the nonlinearity. We finally discuss some examples and special cases to illustrate the results and their application.

\subsection{Preliminaries}

Given $(M, g)$ a complete Riemannian manifold, $w>0$ a smooth positive weight on $M$ with potential $f=-\log w$, the triplet $\left(M, g, e^{-f} d v_{g}\right)$ is referred to as a weighted manifold or a smooth metric measure space (some authors also use the term a manifold with density). The weighted Laplacian is a natural generalisation of the Laplace-Beltrami operator to this weighted context with the two operators coinciding precisely when the potential $f$ is a constant. The weighted Laplacian, denoted $\Delta_{f}$, is a symmetric diffusion operator with respect to the invariant measure $d \mu=e^{-f} d v_{g}$ and as one easily verifies, for $\phi, \psi \in \mathscr{C}_{0}^{\infty}(M)$ :

$$
\int_{M} e^{-f} \psi \Delta_{f} \phi d v_{g}=\int_{M}-e^{-f}\langle\nabla \phi, \nabla \psi\rangle d v_{g}=\int_{M} e^{-f} \phi \Delta_{f} \psi d v_{g}
$$


The symmetric diffusion process $X_{t}$ associated with the weighted Laplacian $\Delta_{f}$ can be constructed by using Itô calculus through the SDE,

$$
d X_{t}=\sqrt{2} d W_{t}-\nabla f\left(X_{t}\right) d t,
$$

where $W_{t}$ is the Riemannian Brownian motion on $M$. The transition probability density function of this diffusion process $X_{t}$ is then exactly the fundamental solution to the weighted heat equation on $M$ :

$$
\square u=\left(\partial_{t}-\Delta_{f}\right) u=0,
$$

i.e., the case $q \equiv 0, \Gamma \equiv 0$ in Eq. 1.1 (see [4-6, 23] for more in this direction).

As for the geometry and curvature properties of the triplet $(M, g, d \mu)$ one introduces for any $m \geq n$ the generalised Bakry-Émery $m$-Ricci curvature tensor (cf. [4-6] for introduction, background and further discussion) by setting 1

$$
\mathscr{R} i c_{f}^{m}(g)=\mathscr{R} i c(g)+\operatorname{Hess}(f)-\frac{\nabla f \otimes \nabla f}{m-n} .
$$

As is readily seen the notion is a generalisation of the Ricci curvature tensor $\mathscr{R} i c=$ $\mathscr{R} i c(g)$ with the influence of $f$ coming through its Hessian and a symmetric 2-tensor built out of its gradient respectively. Notice that when $m=n$ one interprets Eq. 1.7 by admitting only constant functions $f$ in which case $\mathscr{R} i c_{f}^{m}(g) \equiv \mathscr{R} i c(g)$. [Incidentally, the latter relation is true for any $m \geq n$ and $f$ constant]. On the other hand, when $m=\infty$, one simply sets $\mathscr{R} i c_{f}(g):=\mathscr{R} i c_{f}^{\infty}(g) \equiv \mathscr{R} i c(g)+\operatorname{Hess}(f)$ which can be interpreted in a way as the limit $m \nearrow \infty$ in Eq. 1.7. In what follows we shall observe these conventions as regards Eq. 1.7.

For integer $m>n$, the generalised Bakry-Émery $m$-Ricci tensor $\mathscr{R} i c_{f}^{m}$ has a natural geometric interpretation as the Ricci curvature tensor of the warped product metric built out of $g$ on $M \times \mathbb{S}^{m-n}$ (with $\mathbb{S}^{m-n}$ the standard sphere in $\mathbb{R}^{m-n+1}$ ) along horizontal vector fields (see [21, 23, 24]). In many situations this observation gives a direct and natural meaning to geometric quantities and estimates involving this generalised tensor (e.g., in various heat or $W$-entropy formulae). For future reference we recall the weighted Bochner-Weitzenböck formula, taking for smooth functions $u \in \mathscr{C}^{\infty}(M)$ the explicit form,

$$
\frac{1}{2} \Delta_{f}|\nabla u|^{2}=|\operatorname{Hess}(u)|^{2}+\left\langle\nabla u, \nabla \Delta_{f} u\right\rangle+\mathscr{R} i c_{f}(\nabla u, \nabla u) .
$$

A corresponding formula for the generalised Bakry-Émery $m$-Ricci curvature tensor $\mathscr{R} i c_{f}^{m}(g)$ can then be obtained by making use of Eq. 1.7 inside Eq. 1.8 and writing

$$
\frac{1}{2} \Delta_{f}|\nabla u|^{2}=|\operatorname{Hess}(u)|^{2}+\left\langle\nabla u, \nabla \Delta_{f} u\right\rangle+\mathscr{R} i c_{f}^{m}(\nabla u, \nabla u)+\frac{\langle\nabla f, \nabla u\rangle^{2}}{m-n} .
$$

Next through an application of the Cauchy-Schwarz inequality it is easily seen that $(\Delta u)^{2} \leq n|\nabla \nabla u|^{2}$ and subsequently a basic calculation leads to

$$
|\operatorname{Hess}(u)|^{2}+\frac{\langle\nabla f, \nabla u\rangle^{2}}{m-n} \geq \frac{(\Delta u)^{2}}{n}+\frac{\langle\nabla f, \nabla u\rangle^{2}}{m-n} \geq \frac{\left(\Delta_{f} u\right)^{2}}{m} .
$$

As a result replacing in Eq. 1.8 the Hessian term $|\operatorname{Hess}(u)|^{2}=|\nabla \nabla u|^{2}$ with $\left(\Delta_{f} u\right)^{2} / m$ and $\mathscr{R} i c_{f}$ with $\mathscr{R} i c_{f}^{m}$ gives the corresponding inequality

$$
\frac{1}{2} \Delta_{f}|\nabla u|^{2} \geq \frac{1}{m}\left(\Delta_{f} u\right)^{2}+\left\langle\nabla u, \nabla \Delta_{f} u\right\rangle+\mathscr{R} i c_{f}^{m}(\nabla u, \nabla u) .
$$

\footnotetext{
${ }^{1}$ Note that $m$ is not necessarily an integer. In fact for our purposes $m$ is any extended real number verifying $m \geq n$. (See the subsequent discussion.)
} 
A symmetric diffusion operator $L$ is said to satisfy the curvature dimension condition $C D(K, m)$ [for some constant $K \in \mathbb{R}$ and $n \leq m \leq \infty$ ] iff its (iterated) carre du champ operator $\Gamma_{2}[L]$ (as defined in the first identity below) satisfies

$$
\Gamma_{2}[L](u, u):=\frac{1}{2} L|\nabla u|^{2}-\langle\nabla u, \nabla L u\rangle \geq \frac{1}{m}(L u)^{2}+K|\nabla u|^{2},
$$

for every $u \in \mathscr{C}^{\infty}(M)$ [4-6]. In the specific case of the weighted Laplacian $L=\Delta_{f}$ the inequality in Eq. 1.11 shows that

$$
\Gamma_{2}\left[\Delta_{f}\right](u, u)=\frac{1}{2} \Delta_{f}|\nabla u|^{2}-\left\langle\nabla u, \nabla \Delta_{f} u\right\rangle \geq \frac{1}{m}\left(\Delta_{f} u\right)^{2}+\mathscr{R} i c_{f}^{m}(\nabla u, \nabla u) .
$$

Hence a curvature lower bound of the type $\mathscr{R} i c_{f}^{m} \geq K g$ implies the curvature dimension condition $C D(K, m)$ [whilst $\mathscr{R} i c_{f} \geq K g$ implies $C D(K, \infty)$ by Eq. 1.8]. Naturally a lower bound on $\mathscr{R} i c_{f}^{m}$ is a stronger condition than a similar one on $\mathscr{R} i c_{f}$ since from Eq. 1.7 the inequality $R i c_{f}^{m} \geq K g$ implies $R i c_{f} \geq K g$ but not vice versa. For more on consequences of the curvature-dimension condition (e.g., $L^{p}$-boundedness of the $L$ Riesz transforms, Li-Yau estimates, Log Sobolev, isoperimetric, Faber-Krahn and other Sobolev-Poincaré type inequalities) see [6, 12, 23, 29, 36, 42] and the references therein.

Motivated by the above this article makes a contribution by establishing gradient estimates for positive smooth solutions to Eq. 1.1 and the context is one where the metricpotential pair $(g, f)$ are time dependent and form a complete smooth solution (with $0 \leq$ $t \leq T$ for some $T>0)$ to the $(k, m)$-super Perelman-Ricci flow

$$
\left\{\begin{array}{l}
\frac{\partial g}{\partial t}(x, t)+2 \mathscr{R} i c_{f}^{m}(g)(x, t) \geq-2 k g(x, t), \\
\mathscr{R} i c_{f}^{m}(g)(x, t)=\mathscr{R} i c(g)(x, t)+\nabla_{g(t)} \nabla_{g(t)} f(x, t)-\frac{\nabla_{g(t)} f \otimes \nabla_{g(t)} f}{m-n}(x, t) .
\end{array}\right.
$$

It also establishes global estimates with dimension free constants before discussing some implications of these estimates, notably, to parabolic Harnack inequalities and related estimates. In the static case Liouville type results for the elliptic counterpart of Eq. 1.3 are established that include families of Einstein scalar-field and generalised Lichnerowiz type equations (see $[9,11,13,18,30])$. Our results here unifies, extends and improves various earlier results in the literature. Of particular interest here are Liouville theorems for positive bounded solutions to the equation

$$
\mathscr{L}_{\Gamma}(u)=\Delta_{f} u+u \gamma(\log u)+\mathrm{A} u^{\mathrm{p}}+\mathrm{B} u^{\mathrm{q}}=0,
$$

where $\gamma=\gamma(s)$ is a suitable power function, here, primarily and for definiteness, has either forms $s^{\alpha}$ (integer $\alpha \geq 1$ ), $|s|^{\alpha}$ or $|s|^{\alpha-1} s$ (real $\alpha>1$ ) with different sign-changing structures, whilst A, B are suitable constants and p, q are real exponents (see Theorem 4.2). Prompted by the surge of recent interest in such equations we also consider a combined multi-exponent nonlinearity $\Gamma$ and the equation

$$
\mathscr{L}_{\Gamma}(u)=\Delta_{f} u+\sum_{j=1}^{N} a_{j} u^{\mathrm{p}_{j}}+\sum_{j=1}^{N} b_{j} u^{\mathrm{q}_{j}}=0,
$$

with real constants $a_{j}, b_{j}$ and real exponents $\mathrm{p}_{j}, \mathrm{q}_{j}$ (see Theorem 4.3) and closely related to the two families the generalised Lichnerowicz type equations (see Theorem 4.5)

$$
\mathscr{L}_{\Gamma}(u)=\Delta_{f}+\mathrm{A} u^{\mathrm{s}} \log u+\mathrm{B} u^{\mathrm{p}}+\mathrm{C} u^{\mathrm{q}}=0 .
$$

Let us now conclude by giving a quick plan of the paper. The statement and proof of the main estimate appearing in Theorem 2.1 along with the necessary tools span Section 2. 
In the Section 3 we discuss some consequences of Theorem 2.1 that include a parabolic Harnack inequality and a global estimates on positive solutions to Eq. 1.1. Section 4 is devoted to establishing Liouville type theorems on the elliptic counterpart of Eq. 1.1 along with a discussion of the cases described above all under the non-negativity of the generalised Bakry-Émery tensor $\mathscr{R} i c_{f}$ (and $\mathscr{R} i c_{f}^{m}, n \leq m<\infty$ ). Finally Section 5 establishes further global bounds and $p$-estimates on the full evolutionary problem. For background and further discussion relating to the material covered here the reader is referred to the texts $[10,18$, $27,30,34,36,42]$ and the references therein. For recent results and literature on gradient estimates see $[3,8,13,15,19-21,31,35,37,39,40]$ and for closely related topics see [1, $2,7,11,14,16,23,25,26,28,32,38]$.

Notation We write $\square_{q}=\partial_{t}-q-\Delta_{f}$ and $\square=\square_{0}=\partial_{t}-\Delta_{f}$ for the weighted heat operators with or without $q=q(x, t)$ respectively and $\mathscr{L}_{\Gamma}(u)=\Delta_{f} u+\Gamma(u)$. Fixing a reference point $x_{0}$ on $M$ we denote by $d=d_{g(t)}\left(x, x_{0}\right)$ the Riemannian distance between $x_{0}$ and $x$ with respect to $g=g(t)$. At times we write $d=d\left(x, x_{0} ; t\right)$ for simplicity and to avoid having to explicitly write out the metric. In line with common notation we also write $r=r(x, t)$ for the geodesic radial variable expressing the distance from $x$ to $x_{0}$ at time $t$. For $R, T>0$ we introduce the compact space-time set $\mathcal{Q}_{R, T}=\mathcal{Q}_{R, T}\left(x_{0}\right) \equiv\{(x, t)$ : $r(x, t) \leq R, 0 \leq t \leq T\} \subset M \times[0, T]$. We denote by $\mathcal{B}_{R}$ the closed geodesic ball in $M$ with radius $R>0$ and centre $x_{0}$ in the background metric. In addition, we use the symbols $s_{+}=\max (s, 0)$ and $s_{-}=\max (-s, 0)$ for real $s$ when needed and we use $\partial_{t}$ and $\partial / \partial t$ interchangably.

\section{A Local Elliptic Type Gradient Estimate for Eq. 1.1}

We first prove a local elliptic gradient estimate for positive bounded smooth solutions to Eq. 1.1. Here the metric-potential pair is assumed to be a complete smooth solution to the flow inequality Eq. 1.14. This evolution in particular means that the differential operators $\nabla$, div, $\Delta$ and $\Delta_{f}=\Delta-\langle\nabla f, \nabla\rangle$ are time dependent.

For the sake of the estimate below we fix $x_{0} \in M$ and confine to $\mathcal{Q}_{R, T}=\mathcal{Q}_{R, T}\left(x_{0}\right)$ where $R \geq 2, T>0$. The estimate makes use of the bounds on the solution $u$, the lower bound on the Bakry-Émery $m$-Ricci tensor $\mathscr{R} i c_{f}^{m} \geq-(m-1) \mathrm{kg}$ or $\mathscr{R} i c_{f} \geq-(n-1) \mathrm{kg}$ depending on $m \in[n, \infty]$ as needed, and the lower bound $\partial_{t} g \geq-2 \mathrm{~h} g$, with $\mathrm{k}, \mathrm{h} \geq 0$, all within the compact set $\mathcal{Q}_{R, T}$. To avoid separating the cases $n \leq m<\infty$ and $m=\infty$ and for the sake of uniformity in notation we write $\mathscr{R} i c_{f}^{m} \geq-\mathrm{k}_{m} g$ where $\mathrm{k}_{m}=(m-1) \mathrm{k}$ in the former case and $\mathrm{k}_{\infty}=(n-1) \mathrm{k}$ in the latter.

An important quantity appearing in the estimate is $\mathrm{M}_{\Gamma}=\mathrm{M}_{\Gamma}(u)$ [see Eq. 2.5] directly linked to the nonlinearity $\Gamma$ and the solution $u$ and one that will play a key role later in the Liouville theorems. Note that as $u>0$ and $\mathcal{Q}_{R, T}$ is compact, $u$ is bounded away from zero and from above; hence this quantity is finite. The other quantities appearing here are $\mathrm{H}=\sqrt{\mathrm{k}^{2}+\mathrm{h}^{2}},\left[\gamma_{\Delta_{f}}\right]_{+}$and $\mathrm{N}_{q}$ defined in Eqs. $2.2-2.4$ respectively.

Note that as is evident from Eq. 1.7 a lower bound on $\operatorname{Ric}_{f}(g)$ is a weaker assumption than one on $\operatorname{Ric}_{f}^{m}(g)$ for $m<\infty$ by virtue of $\operatorname{Ric}_{f}^{m} \geq \mathrm{kg}$ implying Ric $\mathrm{C}_{f} \geq \mathrm{kg}$ but not vice versa. It is for this reason that our estimate Eq. 2.1 in the former case [with given lower bound on $\operatorname{Ric}_{f}^{m}(g)$ ] can be given a stronger form as indicated in the final statement of Theorem 2.1. (For further discussion and comments see the remark following the statement of the theorem below.) Finally note that due to the local nature of the estimate here all one needs is for $u$ to be a positive solution in an open set containing $\mathcal{Q}_{R, T}$. 
Theorem 2.1 Let $u$ be a positive bounded solution to Eq. 1.1 verifying $0<u \leq D$ in $\mathcal{Q}_{R, T}$. Suppose $\mathscr{R} i c_{f}^{m}(g) \geq-\mathrm{k}_{m} g$ and $\partial_{t} g \geq-2 \mathrm{~h} g$ in $\mathcal{Q}_{R, T}$ for some $\mathrm{k}_{m}, \mathrm{~h} \geq 0, R \geq 2$ and $T>0$. Then there exists a constant $C>0$ depending only on $n$ and $m$ such that for every $(x, t) \in \mathcal{Q}_{R / 2, T}$ with $t>0$ we have,

$$
\frac{|\nabla u|}{u} \leq C\left\{\frac{1}{\sqrt{t}}+\sqrt{\mathrm{H}}+\frac{1}{R}+\sqrt{\frac{\left[\gamma_{\left.\Delta_{f}\right]_{+}}\right.}{R}}+\mathrm{N}_{q}+\sqrt{\mathrm{M}_{\Gamma}(u)}\right\}\left(1-\log \frac{u}{D}\right) .
$$

Here the terms on the right are given by

$$
\begin{array}{r}
\gamma_{\Delta_{f}}=\max _{(x, t)}\left\{\Delta_{f} r(x, t): d\left(x, x_{0} ; t\right)=1,0 \leq t \leq T\right\}, \\
{\left[\gamma_{\Delta_{f}}\right]_{+}=\max \left(\gamma_{\Delta_{f}}, 0\right), \quad \mathrm{H}=\sqrt{\mathrm{h}^{2}+\mathrm{k}^{2}},}
\end{array}
$$

where $\mathrm{k}$ and $\mathrm{k}_{m}$ are as related above whilst

$$
\begin{gathered}
\mathrm{N}_{q}=\sup _{\mathcal{Q}_{R, T}}\left[q_{+}^{1 / 2}+|\nabla q|^{1 / 3}\right], \quad q_{+}=q_{+}(x, t)=[q(x, t)]_{+}, \\
\mathrm{M}_{\Gamma}(u)=\sup _{\mathcal{Q}_{R, T}}\left\{\left[\frac{u \Gamma^{\prime}(u)+\log (u / D)\left[\Gamma(u)-u \Gamma^{\prime}(u)\right]}{u[1-\log (u / D)]}\right]_{+}\right\} .
\end{gathered}
$$

Moreover when $m<\infty$ the term $\left(\left[\gamma_{\Delta_{f}}\right]_{+} / R\right)^{1 / 2}$ on the right in Eq. 2.1 can be discarded.

Remark 2.2 Let us make a few useful comments on the theorem, its assumptions and proof. First $\mathscr{R} i c_{f}^{m}(g) \geq-\mathrm{k}_{m} g$ and $\partial_{t} g \geq-2 \mathrm{~h} g$ in $\mathcal{Q}_{R, T}$ give Eq. 1.14 with $k=\mathrm{k}_{m}+\mathrm{h} \geq 0$. In the proof the lower bound on $\mathscr{R} i c_{f}^{m}(g)$ is utilised in the application of the generalised Laplacian comparison theorem and the bound $\partial_{t} g \geq-2 \mathrm{~h} g$ is used for controlling the time derivative of the geodesic distance function: $\partial_{t} d=\partial\left[d_{g(t)}\left(x, x_{0}\right)\right] / \partial t$. Both these estimates arise in the localisation stage in the later part of the proof. In passing we point out that whilst the estimate above is of a local nature, we present later on, among other things, global gradient estimates (with a different set of ideas and assumptions), that nicely complement Theorem 2.1 (see, e.g., Theorem 3.3 below as well as Section 5).

In the static case $\partial_{t} g \equiv 0$ and $\partial_{t} f \equiv 0$ and $\mathscr{R} i c_{f}^{m}(g) \geq-\mathrm{k}_{m} g$ we can set $\mathrm{h}=0$ and so $\mathrm{H}=\mathrm{k}$ (this is certainly a solution to the second equation in Eq. 1.1 with $k=\mathrm{k}_{m}$ ). In this case $\gamma_{\Delta_{f}}=\max \left\{\Delta_{f} r(x): d\left(x, x_{0}\right)=1\right\}$ and evidently $\partial_{t} d=\partial\left[d_{g(t)}\left(x, x_{0}\right)\right] / \partial t \equiv$ 0 . This way Theorem 2.1 can also be seen as giving local gradient estimates for positive bounded solutions to Eq. 1.1 on non-evolving (static) weighted manifolds which of course is of independent interest. We discuss the static case more in Section 4.

Before embarking on the proof of Theorem 2.1 we need to establish an auxiliary parabolic inequality (see Lemma 2.4). Later on we establish a localised version of this inequality from which the desired estimate follows by way of suitably controlling all the arising terms. In the following lemma $u$ is taken a solution to Eq. 1.1 but no restriction is made on the evolution of the metric-potential pair beyond usual smoothness. 
Lemma 2.3 Let $u$ be a positive solution to Eq. 1.1 satisfying the bound $0<u \leq D$ and suppose the metric-potential pair is time dependent and of class $\mathscr{C}^{2}$. Put $h=\log (u / D)$ and $w=|\nabla \log (1-h)|^{2}$. Then $w$ satisfies the parabolic identity

$$
\begin{aligned}
\square w= & -\frac{\left[\partial_{t} g+2 \mathscr{R} i c_{f}\right]}{(1-h)^{2}}+\frac{2\langle\nabla h, \nabla q\rangle}{(1-h)^{2}}+\frac{2 q w}{1-h}-2(1-h) w^{2} \\
& -\frac{2 h\langle\nabla h, \nabla w\rangle}{1-h}-2\left|\frac{\nabla^{2} h}{1-h}+\frac{\nabla h \otimes \nabla h}{(1-h)^{2}}\right|^{2} \\
& +2 w\left[\Gamma^{\prime}\left(D e^{h}\right)+\frac{h}{1-h} \frac{\Gamma\left(D e^{h}\right)}{D e^{h}}\right] .
\end{aligned}
$$

Here the tensors $\partial_{t} g$ and $\mathscr{R} i c_{f}$ act on $(\nabla h, \nabla h)$ which is omitted for brevity.

Proof Letting $h=\log (u / D)$ and $w=|\nabla h|^{2} /(1-h)^{2}$ we firstly note that $h \leq 0$ and secondly that

$$
\square h=\left(\partial_{t}-\Delta_{f}\right) h=|\nabla h|^{2}+D^{-1} e^{-h} \Gamma\left(D e^{h}\right)+q .
$$

To calculate $\square w$, it is easily seen that $\nabla w=\nabla|\nabla h|^{2} /(1-h)^{2}+2|\nabla h|^{2} \nabla h /(1-h)^{3}$ and subsequently, by recalling $\Delta_{f} w=\Delta w-\langle\nabla f, \nabla w\rangle$, it follows that

$$
\Delta_{f} w=\frac{\Delta_{f}|\nabla h|^{2}}{(1-h)^{2}}+\frac{4\left\langle\nabla|\nabla h|^{2}, \nabla h\right\rangle}{(1-h)^{3}}+\frac{2|\nabla h|^{2} \Delta_{f} h}{(1-h)^{3}}+\frac{6|\nabla h|^{4}}{(1-h)^{4}} .
$$

Next, the identity $\partial_{t} w=\partial_{t}|\nabla h|^{2} /(1-h)^{2}+2|\nabla h|^{2} \partial_{t} h /(1-h)^{3}$ along with $\partial_{t}|\nabla h|^{2}=$ $-\left[\partial_{t} g\right](\nabla h, \nabla h)+2\left\langle\nabla h, \nabla \partial_{t} h\right\rangle$ together give

$$
\partial_{t} w=-\frac{\left[\partial_{t} g\right](\nabla h, \nabla h)}{(1-h)^{2}}+\frac{2\left\langle\nabla h, \nabla \partial_{t} h\right\rangle}{(1-h)^{2}}+\frac{2|\nabla h|^{2} \partial_{t} h}{(1-h)^{3}} .
$$

Here by referring to the second term on the right and by recalling the evolution of $h$ as per Eq. 2.7 it is seen that

$$
\begin{aligned}
\left\langle\nabla h, \nabla \partial_{t} h\right\rangle= & \left\langle\nabla h, \nabla\left[\Delta_{f} h+|\nabla h|^{2}+D^{-1} e^{-h} \Gamma\left(D e^{h}\right)+q\right\rangle\right. \\
= & \left\langle\nabla h, \nabla \Delta_{f} h\right\rangle+\left\langle\nabla h, \nabla|\nabla h|^{2}\right\rangle \\
& +\langle\nabla h, \nabla q\rangle+|\nabla h|^{2}\left[\Gamma^{\prime}\left(D e^{h}\right)-D^{-1} e^{-h} \Gamma\left(D e^{h}\right)\right] .
\end{aligned}
$$

Therefore by substituting Eq. 2.10 back into Eq. 2.9 we are led to

$$
\begin{aligned}
\partial_{t} w= & -\frac{\left[\partial_{t} g\right](\nabla h, \nabla h)}{(1-h)^{2}}+\frac{2\left\langle\nabla h, \nabla \Delta_{f} h\right\rangle}{(1-h)^{2}}+\frac{2\left\langle\nabla h, \nabla|\nabla h|^{2}\right\rangle}{(1-h)^{2}} \\
& +\frac{2\langle\nabla h, \nabla q\rangle}{(1-h)^{2}}+\frac{2|\nabla h|^{2} \Gamma^{\prime}\left(D e^{h}\right)}{(1-h)^{2}}-\frac{2|\nabla h|^{2} \Gamma\left(D e^{h}\right)}{D e^{h}(1-h)^{2}} \\
& +\frac{2|\nabla h|^{2} \Delta_{f} h}{(1-h)^{3}}+\frac{2|\nabla h|^{4}}{(1-h)^{3}}+\frac{2|\nabla h|^{2} q}{(1-h)^{3}}+\frac{2|\nabla h|^{2} \Gamma\left(D e^{h}\right)}{D e^{h}(1-h)^{3}} .
\end{aligned}
$$


Putting Eq. 2.8 and Eq. 2.11 together and simplifying and rearranging terms then gives

$$
\begin{aligned}
\square w= & -\frac{\left[\partial_{t} g\right](\nabla h, \nabla h)}{(1-h)^{2}}-\frac{\Delta_{f}|\nabla h|^{2}}{(1-h)^{2}}+\frac{2\left\langle\nabla h, \nabla \Delta_{f} h\right\rangle}{(1-h)^{2}}+\frac{2\langle\nabla h, \nabla q\rangle}{(1-h)^{2}} \\
& +\frac{2\left\langle\nabla h, \nabla|\nabla h|^{2}\right\rangle}{(1-h)^{2}}-\frac{4\left\langle\nabla|\nabla h|^{2}, \nabla h\right\rangle}{(1-h)^{3}}+\frac{2|\nabla h|^{4}}{(1-h)^{3}}-\frac{6|\nabla h|^{4}}{(1-h)^{4}} \\
& +\frac{2|\nabla h|^{2} q}{(1-h)^{3}}+\frac{2|\nabla h|^{2} \Gamma^{\prime}\left(D e^{h}\right)}{(1-h)^{2}}-\frac{2|\nabla h|^{2} \Gamma\left(D e^{h}\right)}{D e^{h}(1-h)^{2}}+\frac{2|\nabla h|^{2} \Gamma\left(D e^{h}\right)}{D e^{h}(1-h)^{3}} .
\end{aligned}
$$

By recalling Eq. 1.7 and invoking the weighted Bochner-Weitzenböck formula Eq. 1.8 it is next seen that

$$
\begin{aligned}
\square w= & -\frac{\left[\partial_{t} g+2 \mathscr{R} i c_{f}\right]}{(1-h)^{2}}-\frac{2|\nabla \nabla h|^{2}}{(1-h)^{2}}+\frac{2\left\langle\nabla h, \nabla|\nabla h|^{2}\right\rangle}{(1-h)^{2}} \\
& -\frac{4\left\langle\nabla|\nabla h|^{2}, \nabla h\right\rangle}{(1-h)^{3}}+\frac{2|\nabla h|^{4}}{(1-h)^{3}}-\frac{6|\nabla h|^{4}}{(1-h)^{4}} \\
& +\frac{2|\nabla h|^{2} q}{(1-h)^{3}}+\frac{2\langle\nabla h, \nabla q\rangle}{(1-h)^{2}}+\frac{2|\nabla h|^{2} \Gamma^{\prime}\left(D e^{h}\right)}{(1-h)^{2}} \\
& -\frac{2|\nabla h|^{2} \Gamma\left(D e^{h}\right)}{D e^{h}(1-h)^{2}}+\frac{2|\nabla h|^{2} \Gamma\left(D e^{h}\right)}{D e^{h}(1-h)^{3}} \\
= & -\frac{\left[\partial_{t} g+2 \mathscr{R} i c_{f}\right]}{(1-h)^{2}}-\frac{2}{(1-h)^{2}}\left|\nabla \nabla h+\frac{\nabla h \otimes \nabla h}{(1-h)}\right|^{2} \\
& -\frac{2|\nabla h|^{4}}{(1-h)^{3}}+\frac{2\langle\nabla h, \nabla q\rangle}{(1-h)^{2}+\frac{2|\nabla h|^{2} q}{(1-h)^{3}}} \\
& +\frac{\left\langle 2 \nabla h, \nabla|\nabla h|^{2}\right\rangle}{(1-h)^{2}}+\frac{4|\nabla h|^{4}}{(1-h)^{3}}-\frac{\left\langle 2 \nabla|\nabla h|^{2}, \nabla h\right\rangle}{(1-h)^{3}} \\
& -\frac{4|\nabla h|^{4}}{(1-h)^{4}}+2 D^{-1} \frac{e^{-h|\nabla h|^{2}}}{(1-h)^{2}}\left[D e^{h} \Gamma^{\prime}\left(D e^{h}\right)+\frac{h}{1-h} \Gamma\left(D e^{h}\right)\right] .
\end{aligned}
$$

Transforming back to $w$, noting $\langle(1-h) \nabla h,(1-h) \nabla w\rangle=\left\langle\nabla|\nabla h|^{2}, \nabla h\right\rangle+2|\nabla h|^{4} /(1-h)$ and some straightforward calculations result in the desired conclusion and completes the proof.

Lemma 2.4 Under the assumptions of the previous lemma and Eq. 1.14 the function $w=$ $|\nabla \log (1-h)|^{2}$ with $h=\log (u / D)$ satisfies the parabolic inequality

$$
\begin{aligned}
\square w=\left(\partial_{t}-\Delta_{f}\right) w \leq & -2(1-h) w^{2}+2 k w-\frac{2 h\langle\nabla h, \nabla w\rangle}{1-h}+\frac{2\langle\nabla h, \nabla q\rangle}{(1-h)^{2}} \\
& +\frac{2 q w}{1-h}+2 D^{-1} e^{-h} w\left[D e^{h} \Gamma^{\prime}\left(D e^{h}\right)+\frac{h}{1-h} \Gamma\left(D e^{h}\right)\right] .
\end{aligned}
$$

Proof This is an immediate consequence of Lemma 2.3, the flow inequality Eq. 1.14 and the obvious relation between the Bakry-Émery tensors $\mathscr{R} i c_{f}$ and $\mathscr{R} i c_{f}^{m}$.

We are now in a position to return to Theorem 2.1 and finalise the proof of estimate Eq. 2.1. In doing so we will combine Lemma 2.4 with a space-time localisation argument by 
constructing suitable cut-off functions (see Lemma 2.5). The proof then proceeds by making use of certain geometric inequalities, a weighted Laplacian comparison theorem relying purely on the lower bound of the Bakry-Émery generalised Ricci tensor and a localised maximum principle.

The following lemma provides the necessary apparatus for the construction of smooth space-time cut-off functions. For a proof and related discussion see [3, 8, 30, 31, 39, 41]. (See also [11, 18, 33, 34] and the references therein for more on local estimates in PDE and related techniques.)

Lemma 2.5 Given $R, T>0$ and $\tau \in(0, T]$ there exists a smooth function $\bar{\psi}:[0, \infty) \times$ $[0, T] \rightarrow \mathbb{R}$ such that the following properties hold:

1. $\operatorname{supp} \bar{\psi}(r, t) \subset[0, R] \times[0, T]$ and $0 \leq \bar{\psi}(r, t) \leq 1$ in $[0, R] \times[0, T]$.

2. $\bar{\psi}=1$ in $[0, R / 2] \times[\tau, T]$ and $\partial \bar{\psi} / \partial r=0$ in $[0, R / 2] \times[0, T]$.

3. $|\partial \bar{\psi} / \partial t| \leq c \bar{\psi}^{1 / 2} / \tau$ on $[0, \infty) \times[0, T]$ for some $c>0$ and $\bar{\psi}(r, 0)=0 \forall r \in[0, \infty)$.

4. $-c_{\varepsilon} \bar{\psi}^{\varepsilon} / R \leq \partial \bar{\psi} / \partial r \leq 0$ and $\left|\partial^{2} \bar{\psi} / \partial r^{2}\right| \leq c_{\varepsilon} \bar{\psi}^{\varepsilon} / R^{2}$ hold on $[0, \infty) \times[0, T]$ for every $0<\varepsilon<1$ and some $c_{\varepsilon}>0$.

With the above lemma at our disposal let us now proceed by fixing for $R, T>0$ and $\tau \in(0, T]$ a smooth cut-off function $\psi(x, t)=\bar{\psi}(r(x, t), t)$ supported in the compact set $\mathcal{Q}_{R, T} \subset M \times[0, T]$. Recall that $r(x, t)=d\left(x, x_{0} ; t\right)$ denotes the geodesic radial variable at time $t$. The following is an easy and useful lemma. It will be applied to the localised function $\psi w$ where $w$ and $\psi$ are as in Lemmas 2.3 and 2.5 respectively.

Lemma 2.6 For any pair of smooth functions $\psi$ and $w$ the product $\psi w$ satisfies the parabolic differential relation

$$
2\langle\nabla \psi, \nabla(\psi w)-w \nabla \psi\rangle=(\psi \square w-\square(\psi w)+w \square \psi) \psi
$$

Proof A straightforward calculation gives $\Delta_{f}(\psi w)=\psi \Delta_{f} w+2\langle\nabla \psi, \nabla w\rangle+w \Delta_{f} \psi$ and thus $\left(\partial_{t}-\Delta_{f}\right)(\psi w)=\psi\left(\partial_{t}-\Delta_{f}\right) w-2\langle\nabla \psi, \nabla w\rangle+w\left(\partial_{t}-\Delta_{f}\right) \psi$. The assertion now follows by writing $\psi\langle\nabla \psi, \nabla w\rangle=\langle\nabla \psi, \nabla(\psi w)\rangle-|\nabla \psi|^{2} w$.

Proof (Theorem 2.1) Fix $\tau \in(0, T]$ and consider the function $\psi(x, t)=\bar{\psi}(r(x, t), t)$ with $\bar{\psi}$ as in Lemma 2.5. In what follows we prove that the estimate in the theorem holds for all $(x, \tau)$ satisfying $d\left(x, x_{0} ; \tau\right) \leq R / 2$. The arbitrariness of $\tau$ will then justify the assertion for all $(x, t)$ in $\mathcal{Q}_{R / 2, T}$ with $t>0$. Now moving forward, by making use of the parabolic inequality Eq. 2.14 in Lemmas 2.4 and 2.6 we have,

$$
\begin{aligned}
\square(\psi w) \leq & -\frac{2 h\langle\psi \nabla h, \nabla w\rangle}{1-h}-2(1-h) \psi w^{2}+\frac{2\langle\nabla h, \nabla q\rangle}{(1-h)^{2}} \psi \\
& +\frac{2 q w}{1-h} \psi-\frac{2}{\psi}\langle\nabla \psi, \nabla(\psi w)-w \nabla \psi\rangle \\
& +2\left[\Gamma^{\prime}\left(D e^{h}\right)+\frac{h}{1-h} \frac{\Gamma\left(D e^{h}\right)}{D e^{h}}\right] \psi w \\
& +w \square \psi+2 k \psi w .
\end{aligned}
$$


To proceed forward note firstly that $\langle\psi \nabla h, \nabla w\rangle=\langle\nabla h, \nabla(\psi w)\rangle-\langle\nabla h, \nabla \psi\rangle w$. Therefore by substituting in Eq. 2.16 we have

$$
\begin{aligned}
\square(\psi w) \leq & -\frac{2 h\langle\nabla h, \nabla(\psi w)\rangle}{1-h}+\frac{2 h\langle\nabla h, \nabla \psi\rangle w}{1-h}+\frac{2\langle\nabla h, \nabla q\rangle}{(1-h)^{2}} \psi \\
& +\frac{2 q w}{1-h} \psi-\left\langle\frac{2 \nabla \psi}{\psi}, \nabla(\psi w)-w \nabla \psi\right\rangle-2(1-h) \psi w^{2} \\
& +2\left[\Gamma^{\prime}\left(D e^{h}\right)+\frac{h}{1-h} \frac{\Gamma\left(D e^{h}\right)}{D e^{h}}\right] \psi w \\
& -w \Delta_{f} \psi+w \partial_{t} \psi+2 k \psi w .
\end{aligned}
$$

Suppose that $\psi w$ attains its maximum on $\mathcal{Q}_{R, T}$ at the point $\left(x_{1}, t_{1}\right)$. We can assume without loss of generality that $x_{1}$ is not on the cut-locus of $M$ by Calabi's argument [19]. We also assume that $(\psi w)\left(x_{1}, t_{1}\right)>0$ as otherwise the desired estimate becomes trivial with $w(x, \tau) \leq 0$ whenever $d\left(x, x_{0} ; \tau\right) \leq R / 2$. Thus in particular $t_{1}>0$ by (iii) and at $\left(x_{1}, t_{1}\right)$ we have $\Delta_{f}(\psi w) \leq 0, \partial_{t}(\psi w) \geq 0$ and $\nabla(\psi w)=0$. Hence (2.17) implies after the necessary cancellations that at $\left(x_{1}, t_{1}\right)$ we have

$$
\begin{aligned}
\psi w^{2} \leq & \frac{h\langle\nabla h, \nabla \psi\rangle w}{(1-h)^{2}}+\frac{|\nabla \psi|^{2} w}{\psi(1-h)}+\frac{\langle\nabla h, \nabla q\rangle}{(1-h)^{3}} \psi \\
& +\frac{q w \psi}{(1-h)^{2}}+\left[\Gamma^{\prime}\left(D e^{h}\right)+\frac{h}{1-h} \frac{\Gamma\left(D e^{h}\right)}{D e^{h}}\right] \frac{\psi w}{1-h} \\
& -\frac{w \Delta_{f} \psi}{2(1-h)}+\frac{w \partial_{t} \psi}{2(1-h)}+\frac{k \psi w}{1-h}
\end{aligned}
$$

The aim is now to use Eq. 2.18 to establish the estimate at $(x, \tau)$. Towards this end and prompted by the complexity and the nature of technicalities involved we split the argument by considering two cases separated according as to whether $d\left(x_{1}, x_{0} ; t_{1}\right) \geq 1$ or $d\left(x_{1}, x_{0} ; t_{1}\right) \leq 1$ respectively.

Case 1. Suppose $d\left(x_{1}, x_{0} ; t_{1}\right) \geq 1$. Here we apply properties $(i)$-(iv) in Lemma 2.5, the Cauchy-Schwarz and Young inequalities respectively to obtain suitable upper bounds for each of the nine terms on the right-hand side of (2.18). For the first two terms upon noting the bound $-1<h /(1-h) \leq 0$ we can write

$$
\begin{aligned}
\frac{h\langle\nabla h, \nabla \psi\rangle w}{(1-h)^{2}}+\frac{|\nabla \psi|^{2} w}{\psi(1-h)} & \leq \frac{|h||\nabla h|}{(1-h)^{2}}|\nabla \psi| w+\frac{\sqrt{\psi} w}{1-h} \frac{|\nabla \psi|^{2}}{\psi^{3 / 2}} \\
& \leq \frac{|h| \psi^{3 / 4}}{1-h} w^{3 / 2} \frac{|\nabla \psi|}{\psi^{3 / 4}}+\frac{\sqrt{\psi} w}{1-h} \frac{|\nabla \psi|^{2}}{\psi^{3 / 2}} \\
& \leq \frac{\psi w^{2}}{5}+C \frac{|\nabla \psi|^{4}}{\psi^{3}} \leq \frac{\psi w^{2}}{5}+\frac{C}{R^{4}}
\end{aligned}
$$

For the next pair of terms involving $q$, recalling that $q_{+}=q_{+}(x, t)=[q(x, t)]_{+}$and by writing $\mathrm{N}_{q}=\sup _{\mathcal{Q}_{R, T}}\left[|\nabla q|^{1 / 3}+q_{+}^{1 / 2}\right]$ it follows after making use of the Cauchy-Schwarz 
and Young inequality and the bound $1 /(1-h) \leq 1$ that

$$
\begin{aligned}
\frac{\langle\nabla h, \nabla q\rangle}{(1-h)^{3}} \psi+\frac{w \psi q}{(1-h)^{2}} & \leq \frac{|\langle\nabla h, \nabla q\rangle|}{(1-h)^{3}} \psi+\frac{w \psi q_{+}}{(1-h)^{2}} \\
& \leq \psi^{1 / 4} \sqrt{w} \frac{|\nabla q| \psi^{3 / 4}}{(1-h)^{2}}+\frac{\sqrt{\psi} w q_{+}}{(1-h)^{2}} \sqrt{\psi} \\
& \leq \frac{1}{5} \psi w^{2}+C\left[|\nabla q|^{4 / 3}+q_{+}^{2}\right] \\
& \leq \frac{1}{5} \psi w^{2}+C \sup _{\mathcal{Q}_{R, T}}\left[|\nabla q|^{4 / 3}+q_{+}^{2}\right] \leq \frac{1}{5} \psi w^{2}+C \mathrm{~N}_{q}^{4},
\end{aligned}
$$

by virtue of the basic inequalities

$$
\sup _{\mathcal{Q}_{R, T}}\left[|\nabla q|^{4 / 3}+q_{+}^{2}\right] \leq \sup _{\mathcal{Q}_{R, T}}\left[|\nabla q|^{1 / 3}+q_{+}^{1 / 2}\right]^{4} \leq\left(\sup _{\mathcal{Q}_{R, T}}\left[|\nabla q|^{1 / 3}+q_{+}^{1 / 2}\right]\right)^{4} .
$$

For the $\Gamma$ terms upon setting $\mathrm{M}_{\Gamma}=\sup _{\mathcal{Q}_{R, T}}\left\{\Gamma^{\prime}\left(D e^{h}\right)+\left[h \Gamma\left(D e^{h}\right)\right] /\left[D e^{h}(1-h)\right]\right\}_{+}$we can write

$$
\left[\Gamma^{\prime}\left(D e^{h}\right)+\frac{h}{1-h} \frac{\Gamma\left(D e^{h}\right)}{D e^{h}}\right] \frac{\psi w}{1-h} \leq\left[\Gamma^{\prime}\left(D e^{h}\right)+\frac{h}{1-h} \frac{\Gamma\left(D e^{h}\right)}{D e^{h}}\right]_{+} \frac{\sqrt{\psi} w}{1-h} \leq \frac{\psi w^{2}}{5}+C \mathrm{M}_{\Gamma}^{2}
$$

For the last three terms involving $\square \psi+2 k \psi$ we proceed by bounding each separately by using different sets of ideas as follows. Firstly by recalling that $k=\mathrm{h}+(m-1) \mathrm{k} \geq 0$ (or $k=\mathrm{h}+(n-1) \mathrm{k} \geq 0)$ we can write

$$
\frac{k \psi w}{1-h} \leq \frac{\sqrt{\psi} w}{1-h} k_{+} \sqrt{\psi} \leq \frac{\psi w^{2}}{15}+C k_{+}^{2}=\frac{\psi w^{2}}{15}+C k^{2} .
$$

Next for the remaining terms involving the action by the weighted heat operator on $\psi$, specifically,

$$
\frac{w \square \psi}{2(1-h)}=\frac{w\left[-\Delta_{f} \psi+\partial_{t} \psi\right]}{2(1-h)}
$$

we proceed by splitting and handling the space and time derivatives separately. Indeed to bound the term involving $\Delta_{f} \psi$ we use the generalised Laplacian comparison theorem utilising the lower bound on $\mathscr{R} i c_{f}^{m}(g)$ ([38]). To this end we treat the cases $n \leq m<\infty$ and $m=\infty$ separately. Recall that $r(x, t)=d\left(x, x_{0} ; t\right)$ and here $1 \leq d\left(x_{1}, x_{0} ; t_{1}\right) \leq R$, whilst for the sake of convenience we hereafter write $\bar{\psi}_{r}=\partial_{r} \bar{\psi}, \bar{\psi}_{r r}=\partial_{r}^{2} \bar{\psi}$ and $\bar{\psi}_{t}=\partial_{t} \bar{\psi}$. We also note that although a lower bound on $\mathscr{R} i c_{f}$ is a weaker assumption than a lower bound on $\mathscr{R} i c_{f}^{m}$ and so the conclusion under the latter follows from the former, below, we obtain a slightly sharper estimate in the latter case by making no use of the functional $f$-Laplacain term $\left[\gamma_{\Delta_{f}}\right]_{+}$.

Step 1. $(n \leq m<\infty)$ Here $\Delta_{f} r \leq(m-1) \sqrt{\mathrm{k}} \operatorname{coth}(\sqrt{\mathrm{k}} r)$ by the generalised Laplacian comparison theorem and the bound $\mathscr{R} i c_{f}^{m}(g) \geq-(m-1) \mathrm{kg}$ with $\mathrm{k} \geq 0$ (see [38]). Now since $\psi=\bar{\psi}(r(x, t), t)$ we have $\Delta_{f} \psi=\bar{\psi}_{r r}|\nabla r|^{2}+\bar{\psi}_{r} \Delta_{f} r$ and so by taking into account $\bar{\psi}_{r} \leq 0$ it follows from the upper bound on $\Delta_{f} r$ that

$$
\Delta_{f} \psi \geq \bar{\psi}_{r r}+(m-1) \bar{\psi}_{r} \sqrt{\mathrm{k}} \operatorname{coth}(\sqrt{\mathrm{k}} r)
$$

Next noting $\operatorname{coth}(\sqrt{\mathrm{k}} r) \leq \operatorname{coth}(\sqrt{\mathrm{k}} R / 2)$ and $\sqrt{\mathrm{k}} \operatorname{coth}(\sqrt{\mathrm{k}} R / 2) \leq(2 / R+\sqrt{\mathrm{k}})$ subject to $R / 2 \leq r \leq R$ (the first inequality uses the monotonicity of coth $x$ and the second inequality 
uses the bound $x \operatorname{coth} x \leq 1+x$ for $x>0$ ) we deduce by recalling $\bar{\psi}_{r} \equiv 0$ for $0 \leq r \leq R / 2$ that $(m-1) \bar{\psi}_{r} \sqrt{\mathrm{k}} \operatorname{coth}(\sqrt{\mathrm{k}} r) \geq(m-1) \bar{\psi}_{r}(2 / R+\sqrt{\mathrm{k}})$. Hence

$$
\begin{aligned}
-\Delta_{f} \psi & \leq-\left[\bar{\psi}_{r r}+(m-1) \bar{\psi}_{r} \sqrt{\mathrm{k}} \operatorname{coth}(\sqrt{\mathrm{k}} R / 2)\right] \\
& \leq\left|\bar{\psi}_{r r}\right|+(m-1)(2 / R+\sqrt{\mathrm{k}})\left|\bar{\psi}_{r}\right| .
\end{aligned}
$$

As a result proceeding onto bounding the $\Delta_{f} \psi$ term in Eq. 2.18 here we end up with

$$
\begin{aligned}
-\frac{w \Delta_{f} \psi}{2(1-h)} & =\frac{w\left(-\Delta_{f} \psi\right)}{2(1-h)} \leq \frac{\sqrt{\bar{\psi}} w}{2(1-h)}\left[\frac{\left|\bar{\psi}_{r r}\right|}{\sqrt{\bar{\psi}}}+(m-1)(2 / R+\sqrt{\mathrm{k}}) \frac{\left|\bar{\psi}_{r}\right|}{\sqrt{\bar{\psi}}}\right] \\
& \leq \frac{\bar{\psi} w^{2}}{15}+C\left[\frac{\left|\bar{\psi}_{r r}\right|^{2}}{\bar{\psi}}+m^{2}\left(1 / R^{2}+\mathrm{k}\right) \frac{\left|\bar{\psi}_{r}\right|^{2}}{\bar{\psi}}\right] \\
& \leq \frac{\psi w^{2}}{15}+C \frac{m^{2}}{R^{2}} \frac{1+\mathrm{k} R^{2}}{R^{2}} .
\end{aligned}
$$

Step 2. $(m=\infty)$ As $\mathscr{R} i c_{f}(g) \geq-(n-1) \mathrm{kg}$ with $\mathrm{k} \geq 0$ recalling $\left[\gamma_{\Delta_{f}}\right]_{+}=\max \left(\gamma_{\Delta_{f}}, 0\right)$ from Eq. 2.2, Theorem 3.1 in [38] gives $\Delta_{f} r(x, t) \leq\left[\gamma_{\Delta_{f}}\right]_{+}+(n-1) \mathrm{k}(R-1)$ for $r \geq 1$. Now again by virtue of $\psi=\bar{\psi}(r(x, t), t)$ we can write $\Delta_{f} \psi=\bar{\psi}_{r r}|\nabla r|^{2}+\bar{\psi}_{r} \Delta_{f} r$ and as $\bar{\psi}_{r} \leq 0$ this gives $\Delta_{f} \psi \geq \bar{\psi}_{r r}+\bar{\psi}_{r}\left\{\left[\gamma_{\Delta_{f}}\right]_{+}+(n-1) \mathrm{k}(R-1)\right\}[c f$. (ii) and (iv) in Lemma 2.5]. Therefore putting these together results in

$$
\begin{aligned}
-\Delta_{f} \psi & \leq-\bar{\psi}_{r r}-\left\{\left[\gamma_{\Delta_{f}}\right]_{+}+(n-1) \mathrm{k}(R-1)\right\} \bar{\psi}_{r} \\
& \leq\left|\bar{\psi}_{r r}\right|+\left\{\left[\gamma_{\Delta_{f}}\right]_{+}+(n-1) \mathrm{k}(R-1)\right\}\left|\bar{\psi}_{r}\right|,
\end{aligned}
$$

and so moving onto bounding the $\Delta_{f} \psi$ term in Eq. 2.18 in this case we end up with

$$
\begin{aligned}
-\frac{w \Delta_{f} \psi}{2(1-h)} & =\frac{w\left(-\Delta_{f} \psi\right)}{2(1-h)} \leq \frac{\sqrt{\bar{\psi}} w}{2(1-h)}\left[\frac{\left|\bar{\psi}_{r r}\right|}{\sqrt{\bar{\psi}}}+\left\{\left[\gamma_{\Delta_{f}}\right]_{+}+(n-1) \mathrm{k}(R-1)\right\} \frac{\left|\bar{\psi}_{r}\right|}{\sqrt{\bar{\psi}}}\right] \\
& \leq \frac{\bar{\psi} w^{2}}{15}+C\left[\frac{\left|\bar{\psi}_{r r}\right|^{2}}{\bar{\psi}}+\left[\gamma_{\Delta_{f}}\right]_{+}^{2} \frac{\left|\bar{\psi}_{r}\right|^{2}}{\bar{\psi}}+n^{2} \mathrm{k}^{2} R^{2} \frac{\left|\bar{\psi}_{r}\right|^{2}}{\bar{\psi}}\right] \\
& \leq \frac{\psi w^{2}}{15}+C \frac{n^{2}}{R^{2}}\left[\frac{1}{R^{2}}+\left[\gamma_{\Delta_{f}}\right]_{+}^{2}+\mathrm{k}^{2} R^{2}\right] .
\end{aligned}
$$

In order to bound the term involving $\partial_{t} \phi$ we proceed as follows. For $d\left(x_{1}, x_{0} ; t\right) \leq R$ and fixed $t>0$ let $\zeta=\zeta(s):[0, d] \rightarrow M$ be a minimal geodesic with respect to $g(t)$ connecting $x_{0}=\zeta(0)$ to $x_{1}=\zeta(d)$. Then a standard calculation utilising $\partial_{t} g \geq-2 \mathrm{~h} g$ in $\mathcal{Q}_{R, T}$ with $\mathrm{h} \geq 0$ as in the theorem gives

$$
\begin{aligned}
\partial_{t} r\left(x_{1}, t\right) & =\partial_{t} d\left(x_{1}, x_{0} ; t\right)=\partial_{t} \int_{0}^{d}\left|\zeta^{\prime}(s)\right|_{g(t)} d s \\
& =\int_{0}^{d} \frac{\left[\partial_{t} g\right]\left(\zeta^{\prime}, \zeta^{\prime}\right)}{2\left|\zeta^{\prime}\right|_{g(t)}} d s \\
& \geq \int_{0}^{d}-\mathrm{h}\left|\zeta^{\prime}\right|_{g(t)} d s \geq-\mathrm{h} r\left(x_{1}, t\right) \geq-\mathrm{h} R .
\end{aligned}
$$

Now referring to $\psi(x, t)=\bar{\psi}(r(x, t), t)$ and applying the properties of $\bar{\psi}=\bar{\psi}(r, t)$ as listed in Lemma 2.5 we can then write

$$
\partial_{t} \psi=\bar{\psi}_{t}+\bar{\psi}_{r} \partial_{t} r \leq \bar{\psi}_{t}-\mathrm{h} R \bar{\psi}_{r} \leq\left[\frac{\left|\bar{\psi}_{t}\right|}{\sqrt{\bar{\psi}}}+R \frac{\mathrm{h}\left|\bar{\psi}_{r}\right|}{\sqrt{\bar{\psi}}}\right] \sqrt{\bar{\psi}} \leq C(1+\tau \mathrm{h}) \frac{\sqrt{\psi}}{\tau} .
$$


Hence basic considerations lead to the bound

$$
\frac{w \partial_{t} \psi}{2(1-h)}=\frac{\sqrt{\psi} w}{2(1-h)} \frac{\partial_{t} \psi}{\sqrt{\psi}} \leq C \sqrt{\psi} w \frac{1+\tau h}{\tau} \leq \frac{1}{15} \psi w^{2}+C \frac{1+\tau^{2} h^{2}}{\tau^{2}}
$$

Thus, in summary, by referring to the last three terms in Eq. 2.18 we have obtained, subject to $\mathscr{R} i c_{f}^{m}(g) \geq-(m-1) \mathrm{k} g$ and with $k=\mathrm{h}+(m-1) \mathrm{k}$ the bound,

$$
\begin{aligned}
\frac{w(\square+2 k) \psi}{2(1-h)} & =\frac{w\left[-\Delta_{f} \psi+\partial_{t} \psi\right]}{2(1-h)}+\frac{k \psi w}{1-h} \\
& \leq \frac{\phi w^{2}}{5}+C\left[\frac{1}{R^{4}}+\frac{\mathrm{k}}{R^{2}}+\frac{1}{\tau^{2}}+\mathrm{h}^{2}+k^{2}\right] \\
& \leq \frac{\phi w^{2}}{5}+C\left[\frac{1}{R^{4}}+\frac{1}{\tau^{2}}+\mathrm{h}^{2}+\mathrm{k}^{2}\right]
\end{aligned}
$$

using k $/ R^{2} \leq \mathrm{k}^{2} / 2+1 /\left(2 R^{4}\right)$ with $C=C(m)>0$, and subject to $\mathscr{R} i c_{f}(g) \geq-(n-1) \mathrm{kg}$ and with $k=\mathrm{h}+(n-1) \mathrm{k}$ the bound,

$$
\begin{aligned}
\frac{w(\square+2 k) \psi}{2(1-h)} & \leq \frac{\phi w^{2}}{5}+C\left[\frac{1}{R^{4}}+\frac{\left[\gamma_{\Delta_{f}}\right]_{+}^{2}}{R^{2}}+\mathrm{k}^{2}+\frac{1}{\tau^{2}}+\mathrm{h}^{2}+k^{2}\right] \\
& \leq \frac{\phi w^{2}}{5}+C\left[\frac{1}{R^{4}}+\frac{\left[\gamma_{\Delta_{f}}\right]_{+}^{2}}{R^{2}}+\frac{1}{\tau^{2}}+\mathrm{h}^{2}+\mathrm{k}^{2}\right] .
\end{aligned}
$$

with $C=C(n)>0$.

At this stage we have completed estimating each of the nine individual terms on the righthand side of Eq. 2.18. Inserting these back into the inequality and adjusting the constant, we obtain at $\left(x_{1}, t_{1}\right)$, the inequality

$$
\psi w^{2} \leq \frac{4}{5} \psi w^{2}+C\left[\frac{1}{R^{4}}+\frac{\left[\gamma_{\Delta_{f}}\right]_{+}^{2}}{R^{2}}+\frac{1}{\tau^{2}}+\mathrm{H}^{2}+\mathrm{N}_{q}^{4}+\mathrm{M}_{\Gamma}^{2}\right]
$$

where $\mathrm{H}^{2}=\mathrm{h}^{2}+\mathrm{k}^{2}$. Note that referring to the expression on the right here, in the case resulting from Eq. 2.26, the term $\left[\gamma_{\Delta_{f}}\right]_{+}^{2} / R^{2}$ can be discarded as is seen by comparing the bounds on the right-hand side of Eqs. 2.30 and 2.31.

Now using the maximal characterisation of the point $\left(x_{1}, t_{1}\right)$, for any $(x, t) \in \mathcal{Q}_{R, T}$, we have $[\psi w]^{2}(x, t) \leq[\psi w]^{2}\left(x_{1}, t_{1}\right) \leq\left[\psi w^{2}\right]\left(x_{1}, t_{1}\right)$, and so when combined with the above we have at any $(x, t) \in \mathcal{Q}_{R, T}$ the bound

$$
[\psi w]^{2}(x, t)=\frac{\psi^{2}|\nabla h|^{4}}{(1-h)^{4}}(x, t) \leq C\left[\frac{1}{R^{4}}+\frac{\left[\gamma_{\Delta_{f}}\right]_{+}^{2}}{R^{2}}+\frac{1}{\tau^{2}}+\mathrm{H}^{2}+\mathrm{N}_{q}^{4}+\mathrm{M}_{\Gamma}^{2}\right] .
$$

Since $\psi(x, \tau)=1$ for when $d\left(x, x_{0} ; \tau\right) \leq R / 2$, we hence obtain at $(x, \tau)$ the bound

$$
\sqrt{w}=\frac{|\nabla h|}{1-h} \leq C\left[\frac{1}{R}+\sqrt{\frac{\left[\gamma_{\Delta_{f}}\right]_{+}}{R}}+\frac{1}{\sqrt{\tau}}+\sqrt{\mathrm{H}}+\mathrm{N}_{q}+\sqrt{\mathrm{M}_{\Gamma}}\right] .
$$

Case 2. Suppose $d\left(x_{1}, x_{0} ; t_{1}\right) \leq 1$. As by (ii) $\psi$ is a constant function in space direction (when $d\left(x, x_{0} ; t\right) \leq R / 2, t \in[0, T]$ with $R \geq 2$ ), referring to Eq. 2.18, the terms involving 
space derivatives of $\psi$ vanish or largely simplify (in particular $\nabla \psi=0, \Delta_{f} \psi=0$ and $\left.\partial_{t} \psi=\partial_{t} \bar{\psi}\right)$. Therefore it follows that at $\left(x_{1}, t_{1}\right)$, we have

$$
\begin{aligned}
w^{2} & \leq \frac{\langle\nabla h, \nabla q\rangle}{(1-h)^{3}}+\frac{q w}{(1-h)^{2}}+\left[\Gamma^{\prime}\left(D e^{h}\right)+\frac{h}{1-h} \frac{\Gamma\left(D e^{h}\right)}{D e^{h}}\right] \frac{w}{1-h}+\frac{w\left(\partial_{t} \psi+2 k \psi\right)}{2 \psi(1-h)} \\
& \leq \sqrt{w}\left\{\frac{|\nabla q|}{1-h}+\left(\frac{q_{+}}{1-h}+\left[\Gamma^{\prime}\left(D e^{h}\right)+\frac{h}{1-h} \frac{\Gamma\left(D e^{h}\right)}{D e^{h}}\right]_{+}+\frac{\left|\bar{\psi}_{t}\right|}{2 \psi}+k\right) \sqrt{w}\right\} .
\end{aligned}
$$

Therefore basic considerations and an application of Young's inequality give

$$
\begin{aligned}
w^{3 / 2} & \leq|\nabla q|+\left(q_{+}+\mathrm{M}_{\Gamma}+C / \tau+k\right) \sqrt{w} \\
& \leq|\nabla q|+2 / 3\left(q_{+}+\mathrm{M}_{\Gamma}+C / \tau+k\right)^{3 / 2}+w^{3 / 2} / 3,
\end{aligned}
$$

which then imply $w \leq 2\left(|\nabla q|^{2 / 3}+q_{+}+\mathrm{M}_{\Gamma}+C / \tau+k\right)$. As $\psi \equiv 1$, when $d\left(x, x_{0} ; t\right) \leq$ $R / 2$ and $\tau \leq t \leq T$ (thus in particular for $t=\tau$ ) by $(i)$, we have the inequalities $w(x, \tau)=\psi w(x, \tau) \leq \psi w\left(x_{1}, t_{1}\right) \leq w\left(x_{1}, t_{1}\right)$ due to $\psi\left(x_{1}, t_{1}\right) \leq 1, \psi(x, \tau)=1$. Therefore $w(x, \tau) \leq C\left(\mathrm{~N}_{q}^{2}+\mathrm{M}_{\Gamma}+1 / \tau+k\right)$. Hence recalling the definition of $w$ and its relation to $h$, it follows that at $(x, \tau)$ we have

$$
\sqrt{w}=\frac{|\nabla h|}{1-h} \leq C\left[\mathrm{~N}_{q}+\sqrt{\mathrm{M}_{\Gamma}}+\frac{1}{\sqrt{\tau}}+\sqrt{k}\right] .
$$

This together with the arbitrariness of $\tau>0$ leads to a special case of Eq. 2.1. We have thus shown that in either case the estimate holds true and this completes the proof.

Corollary 2.7 Under the assumptions of Theorem 2.1 and the global curvature bounds $\mathscr{R} i c_{f}^{m}(g) \geq-\mathrm{k}_{m} g$ and $\partial_{t} g \geq-2 \mathrm{~h} g$ on $M \times[0, T]$, for any bounded positive solution $u$ to Eq. 1.1 satisfying $0<u \leq D$ on $M$ and $0<t<T$ we have

$$
\frac{|\nabla u|}{u} \leq C\left[\sqrt{\mathrm{H}}+\frac{1}{\sqrt{t}}+\mathrm{N}_{q}+\sqrt{\mathrm{M}_{\Gamma}(u)}\right]\left(1-\log \frac{u}{D}\right) .
$$

Here $\mathrm{N}_{q}, \mathrm{M}_{\Gamma}$ have the same meanings as in Theorem 2.1 except for the supremums that are now taken over $M \times[0, T]$ instead of $\mathcal{Q}_{R, T}$ and assumed to be finite.

Proof By virtue of the estimate being true for every $R \geq 2$, and with the constants being independent or $R$, passing to the limit $R \rightarrow \infty$ gives the desired conclusion.

\section{Two Parabolic Harnack Inequalities}

In this section and next we discuss some consequences of Theorem 2.1 that are of a global nature. Here the emphasis will be on parabolic Harnack inequalities and a global gradient estimate with dimension free constants and in the next section we establish Liouville type theorems and discuss some related examples.

Theorem 3.1 Under the assumptions of Theorem 2.1 and the global curvature bounds $\mathscr{R} i c_{f}^{m}(g) \geq-\mathrm{k}_{m} g$ and $\partial_{t} g \geq-2 \mathrm{~h} g$ on $M \times[0, T]$, for any positive bounded solution $u$ to Eq. 1.1 satisfying $0<u \leq D$ and any $x_{1}, x_{2} \in M$ and $t \in(0, T]$ we have

$$
\frac{u\left(x_{1}, t\right)}{e D} \leq\left[\frac{u\left(x_{2}, t\right)}{e D}\right]^{\Sigma}
$$


where

$$
\Sigma=\exp \left\{-C d\left(x_{1}, x_{2} ; t\right)\left[\sqrt{\mathrm{H}}+\frac{1}{\sqrt{t}}+\mathrm{N}_{q}+\sqrt{\mathrm{M}_{\Gamma}(u)}\right]\right\} .
$$

Proof Pick $x_{1}, x_{2}$ in $M$ and fix $0<t<T$. Let $\zeta=\zeta(s)$ with $0 \leq s \leq 1$ be a shortest curve with respect to $g=g(t)$ joining $x_{1}, x_{2}$ in $M$, specifically, $\zeta(0)=x_{1}$ and $\zeta(1)=x_{2}$. Put $d=d\left(x_{1}, x_{2} ; t\right)$ and assume that $\mathrm{N}_{q}, \mathrm{M}_{\Gamma}<\infty$ (otherwise $\Sigma=0$ and the inequality is trivially true). Next, by utilising Corollary 2.7, recalling $h=\log (u / D)$ and noting the inequality $1-h>0$, we can write

$$
\begin{aligned}
\log \frac{1-h\left(x_{2}, t\right)}{1-h\left(x_{1}, t\right)} & =\int_{0}^{1} \frac{d}{d s} \log [1-h(\zeta(s), t)] d s=\int_{0}^{1}-\frac{\left\langle\nabla h(\zeta(s), t), \zeta^{\prime}(s)\right\rangle}{1-h(\zeta(s), t)} d s \\
& \leq \int_{0}^{1} \frac{|\nabla h|\left|\zeta^{\prime}\right|}{1-h} d s \leq C d\left[\sqrt{\mathrm{H}}+\frac{1}{\sqrt{t}}+\mathrm{N}_{q}+\sqrt{\mathrm{M}_{\Gamma}}\right] .
\end{aligned}
$$

Here we have used $|\nabla h| /(1-h)=|\nabla \log u| /[1-\log (u / D)]$ along with Eq. 2.35. Therefore a direct set of calculations give,

$$
\frac{\log \left[e D / u\left(x_{2}, t\right)\right]}{\log \left[e D / u\left(x_{1}, t\right)\right]}=\frac{1-h\left(x_{2}, t\right)}{1-h\left(x_{1}, t\right)} \leq \exp \left\{C d\left[\sqrt{\mathrm{H}}+\frac{1}{\sqrt{t}}+\mathrm{N}_{q}+\sqrt{\mathrm{M}_{\Gamma}}\right]\right\}
$$

and so the assertion follows.

The next theorem is a global estimate with dimension free constants. Such estimates are of independent interest and have been mainly proved for the heat equation using a variety of methods (see, e.g., [3, 15, 17, 22, 41]). Below we establish one such estimate for the positive solutions to the nonlinear Eq. 1.1. For convenience here we confine to $M$ being closed however point out that extensions to non-compact or other cases is also possible. Before that we have the following lemma which is of independent interest and requires no extra assumptions.

Lemma 3.2 Let $u$ be a bounded positive solution to Eq. 1.1 with $0<u \leq D$ and assume the metric-potential pair evolves under the super Perelman-Ricci flow Eq. 1.14. Let

$$
\mathscr{P}_{\gamma}[u]=\gamma(t)|\nabla u|^{2} / u-u \log (D / u),
$$

where $\gamma$ is a smooth, non-negative but otherwise arbitrary function. Then we have

$$
\begin{aligned}
\square_{q} \mathscr{P}_{\gamma}[u] \leq & 2 \gamma\langle\nabla u, \nabla q\rangle+q u+\left(\gamma^{\prime}+2 k \gamma-1\right) \frac{|\nabla u|^{2}}{u} \\
& +\Gamma(u)+2 \gamma\left[\Gamma^{\prime}(u)-\frac{\Gamma(u)}{u}\right] \frac{|\nabla u|^{2}}{u}+\mathscr{P}_{\gamma}[u] \frac{\Gamma(u)}{u} .
\end{aligned}
$$

Proof We start by evaluating the individual ingredients in the expression on the left-hand side of Eq. 3.6. Towards this end, making note of Eq. 3.5, it is firstly seen that

$$
\begin{aligned}
\partial_{t} \frac{\gamma|\nabla u|^{2}}{u} & =\frac{\gamma^{\prime}|\nabla u|^{2}}{u}+\frac{\gamma \partial_{t}|\nabla u|^{2}}{u}-\frac{\gamma|\nabla u|^{2} \partial_{t} u}{u^{2}} \\
& =\frac{\gamma^{\prime}|\nabla u|^{2}}{u}+\frac{\gamma}{u}\left[-\left[\partial_{t} g\right](\nabla u, \nabla u)+2\left\langle\nabla u, \nabla \partial_{t} u\right\rangle-\frac{|\nabla u|^{2}}{u} \partial_{t} u\right],
\end{aligned}
$$


whilst upon noting $\nabla\left(|\nabla u|^{2} / u\right)=\left[u \nabla|\nabla u|^{2}-|\nabla u|^{2} \nabla u\right] / u^{2}$ and $\Delta_{f} v=\Delta v-\langle\nabla f, \nabla v\rangle$ we have

$$
u^{3} \Delta_{f} \frac{|\nabla u|^{2}}{u}=u^{2} \Delta_{f}|\nabla u|^{2}-2 u\left\langle\nabla|\nabla u|^{2}, \nabla u\right\rangle-u|\nabla u|^{2} \Delta_{f} u+2|\nabla u|^{4} .
$$

Similar calculations give $\partial_{t}[u \log (D / u)]=\partial_{t} u \log (D / u)-\partial_{t} u=[\log (D / u)-1] \partial_{t} u$ and $\Delta_{f}[u \log (D / u)]=[\log (D / u)-1] \Delta_{f} u-|\nabla u|^{2} / u$. Now referring again to Eq. 3.5 and putting together the above fragments it follows that

$$
\begin{aligned}
\square_{q} \mathscr{P}_{\gamma}[u]= & \left(\partial_{t}-q-\Delta_{f}\right) \mathscr{P}_{\gamma}[u]=\frac{\gamma^{\prime}|\nabla u|^{2}}{u}+\gamma \square_{q} \frac{|\nabla u|^{2}}{u}-\square_{q}[u \log (D / u)] \\
= & \frac{\gamma}{u}\left\{2\left\langle\nabla u, \nabla \partial_{t} u\right\rangle-\left[\partial_{t} g\right](\nabla u, \nabla u)-\Delta_{f}|\nabla u|^{2}+\frac{2}{u}\left\langle\nabla|\nabla u|^{2}, \nabla u\right\rangle-\frac{2|\nabla u|^{4}}{u^{2}}\right\} \\
& +\left(\gamma^{\prime}-1\right) \frac{|\nabla u|^{2}}{u}-[\log (D / u)-1] \square_{q} u-\gamma \frac{|\nabla u|^{2}}{u^{2}} \square_{q} u+q u-2 q \frac{\gamma|\nabla u|^{2}}{u} .
\end{aligned}
$$

Therefore upon invoking Eq. 1.1 and subsequently making the substitutions $\square_{q} u=\Gamma(u)$ and $2\left\langle\nabla u, \nabla \partial_{t} u\right\rangle=2\left\langle\nabla u, \nabla \Delta_{f} u\right\rangle+2\langle\nabla u, \nabla[q u+\Gamma(u)]\rangle$ it follows that

$$
\begin{aligned}
\square_{q} \mathscr{P}_{\gamma}[u]= & \frac{\gamma}{u}\left\{-\left[\partial_{t} g\right](\nabla u, \nabla u)+2\left\langle\nabla u, \nabla \Delta_{f} u\right\rangle+2\langle\nabla u, \nabla[q u+\Gamma(u)]\rangle\right. \\
& \left.-\Delta_{f}|\nabla u|^{2}+\frac{2}{u}\left\langle\nabla|\nabla u|^{2}, \nabla u\right\rangle-\frac{2|\nabla u|^{4}}{u^{2}}\right\}+\left(\gamma^{\prime}-1\right) \frac{|\nabla u|^{2}}{u} \\
& -[\log (D / u)-1] \Gamma(u)+q u-\gamma \frac{|\nabla u|^{2}}{u^{2}}[2 q u+\Gamma(u)] .
\end{aligned}
$$

Now making use of the weighted Bochner-Weitzenöck formula Eq. 1.8 followed by other basic considerations we can write

$$
\begin{aligned}
\square_{q} \mathscr{P}_{\gamma}[u]= & -\frac{\gamma}{u}\left[\left[\partial_{t} g\right](\nabla u, \nabla u)+2|\nabla \nabla u|^{2}+2 \mathscr{R} i c_{f}(\nabla u, \nabla u)-\frac{2\left\langle\nabla|\nabla u|^{2}, \nabla u\right\rangle}{u}\right] \\
& -2 \frac{\gamma}{u} \frac{|\nabla u|^{4}}{u^{2}}+\frac{2 \gamma}{u}\langle\nabla u, \nabla(q u)\rangle+\frac{2 \gamma}{u}\langle\nabla u, \nabla \Gamma(u)\rangle+\left(\gamma^{\prime}-1\right) \frac{|\nabla u|^{2}}{u} \\
& -[\log (D / u)-1] \Gamma(u)+q u-\gamma \frac{|\nabla u|^{2}}{u^{2}}[2 q u+\Gamma(u)] .
\end{aligned}
$$

Thus by virtue of $|\nabla \nabla u|^{2}-u^{-1}\left\langle\nabla|\nabla u|^{2}, \nabla u\right\rangle+u^{-2}|\nabla u|^{4}=\left|\nabla^{2} u-u^{-1}[\nabla u \otimes \nabla u]\right|^{2} \geq 0$ and by an application of the inequality $\partial_{t} g+2 \mathscr{R} i c_{f} \geq-2 \mathrm{~kg}$ we arrive at

$$
\begin{aligned}
\square_{q} \mathscr{P}_{\gamma}[u] \leq & 2 \gamma\langle\nabla u, \nabla q\rangle+q u+\left(\gamma^{\prime}+2 k \gamma-1\right) \frac{|\nabla u|^{2}}{u} \\
& +\frac{\gamma|\nabla u|^{2}}{u}\left[2 \Gamma^{\prime}(u)-\frac{\Gamma(u)}{u}\right]+[1-\log (D / u)] \Gamma(u),
\end{aligned}
$$

which after rearranging terms and substituting from Eq. 3.5 gives the desired inequality.

Theorem 3.3 Let $u$ be a positive solution to Eq. 1.1 with $q=0$ satisfying $0<u \leq D$ in $M \times[0, T]$ and assume the metric-potential pair $(g, f)$ evolves under Eq. 1.14 with $k \geq 0$. Suppose $\Gamma(u) \geq 0$ and $\Gamma^{\prime}(u) \leq 0$ along the solution $u$ and that $M$ is closed. Then

$$
t|\nabla \log u|^{2} \leq(1+2 k t)[1+\log (D / u)], \quad 0<t<T .
$$


Moreover, there holds the following interpolation inequality for $\delta>0, x_{1}, x_{2} \in M$

$$
\frac{u\left(x_{1}, t\right)}{e D} \leq \exp \left(\frac{d^{2}\left(x_{1}, x_{2}, t\right)[1+2 k t]}{4 \delta t}\right)\left[\frac{u\left(x_{2}, t\right)}{e D}\right]^{1 /(1+\delta)},
$$

where $t \in(0, T]$.

Proof The function $\gamma(t)=t /(1+2 k t)$ is non-negative and satisfies $\gamma^{\prime}+2 k \gamma-1 \leq 0$. Now consider using Lemma 3.2 with $\tilde{D}=e D$ in place of $D$ there. Evidently $u \leq \tilde{D}$ and referring to Eq. 3.6 and using $q=0$ we have

$$
\begin{aligned}
\square_{q} \mathscr{P}_{\gamma}[u]=\square \mathscr{P}_{\gamma}[u] \leq & 2 \gamma\langle\nabla u, \nabla q\rangle+q u+\left(\gamma^{\prime}+2 k \gamma-1\right) \frac{|\nabla u|^{2}}{u} \\
& +\frac{\gamma|\nabla u|^{2}}{u}\left[2 \Gamma^{\prime}(u)-\frac{\Gamma(u)}{u}\right]+[1-\log (\tilde{D} / u)] \Gamma(u) \\
\leq & 2 \frac{\gamma|\nabla u|^{2}}{u} \Gamma^{\prime}(u)-\frac{\gamma|\nabla u|^{2}}{u^{2}} \Gamma(u)-\log (D / u) \Gamma(u) \leq 0 .
\end{aligned}
$$

Next by an easy inspection $\mathscr{P}_{\gamma}[u](x, 0) \leq 0$ for all $x \in M$. Furthermore as seen above $\square \mathscr{P}_{\gamma}[u]=\left(\partial_{t}-\Delta_{f}\right) \mathscr{P}_{\gamma}[u] \leq 0$. Thus an application of maximum principle (to $\Delta_{f}$ ) gives $\mathscr{P}_{\gamma}[u](x, t) \leq 0$ for all $(x, t)$ in $M \times[0, T]$ from which the desired estimate Eq. 3.12 follows.

Next to prove Eq. 3.13 set $\mathcal{Z}(x, t)=\log [\tilde{D} / u(x, t)]$. Then by (3.12) we obtain

$$
|\nabla \sqrt{\mathcal{Z}}|=\left|\frac{\nabla u / u}{2 \sqrt{\mathcal{Z}}}\right| \leq \frac{\sqrt{1+2 k t}}{2 \sqrt{t}} .
$$

Integrating the above along a minimising geodesic joining a pair of points $x_{1}, x_{2}$ in $M$ then gives

$$
\sqrt{\log (\tilde{D} / u(y, t))} \leq \sqrt{\log (\tilde{D} / u(x, t))}+d(x, y ; t) \frac{\sqrt{1+2 k t}}{2 \sqrt{t}} .
$$

For any $\delta>0$ thus $\log (\tilde{D} / u(y, t)) \leq(1+\delta)\left[\log (\tilde{D} / u(x, t))+d^{2}(x, y ; t)(1+2 k t) /(4 \delta t)\right]$. Exponentiating and rearranging yields the desired inequality Eq. 3.13.

\section{Liouville and Global Constancy Type Results}

Let us now move onto the static case and discuss some implications of Theorem 2.1 in this closely related context. Thus here $\left(M, g, e^{-f} d v\right)$ is a complete weighted manifold with the metric-potential pair $(g, f)$ being time independent and $\mathscr{R} i c_{f}^{m}(g) \geq-\mathrm{k}_{m} g$ in $\mathcal{B}_{R} \subset M$ for some $\mathrm{k}_{m} \geq 0, R \geq 2$ as before. (Note that $\mathcal{B}_{R}$ is the closed geodesic ball in $M$ with radius $R>0$ and centre $x_{0}$.) We consider positive solutions $u=u(x)$ to the elliptic equation $\mathscr{L}_{\Gamma}(u)+q(x) u=\Delta_{f} u+q(x) u+\Gamma(u)=0$ in $\mathcal{B}_{R} \subset M$. As here $u, q$ as well as $(g, f)$ are time independent and the constants do not depend on time we can pass to the limit $t \nearrow \infty$ in Eq. 2.1 and deduce the following local estimate.

Corollary 4.1 Let $\left(M, g, e^{-f} d v_{g}\right)$ be a weighted manifold with Bakry-Émery curvature satisfying the lower bound $\mathscr{R} i c_{f}^{m} \geq-\mathrm{k}_{m}$ g in $\mathcal{B}_{R}$ for some $\mathrm{k}_{m} \geq 0$. Let $u$ be a positive solution to

$$
\mathscr{L}_{\Gamma}(u)+q(x) u=\Delta_{f} u+q(x) u+\Gamma(u)=0,
$$


in $\mathcal{B}_{R}$. Then there exists a constant $C>0$ depending on $n, m$ only such that in $\mathcal{B}_{R / 2}$ we have

$$
\frac{|\nabla u|}{u} \leq C\left[\frac{1}{R}+\sqrt{\frac{\left[\gamma_{\left.\Delta_{f}\right]_{+}}\right.}{R}}+\sqrt{\mathrm{k}}+\mathrm{N}_{q}+\sqrt{\mathrm{M}_{\Gamma}(u)}\right]\left(1-\log \frac{u}{D}\right) .
$$

Here $0<u \leq D,\left[\gamma_{\Delta_{f}}\right]_{+}=\left[\max _{\left\{x: d\left(x, x_{0}\right)=1\right\}} \Delta_{f} r(x)\right]_{+}, \mathrm{N}_{q}=\sup _{\mathcal{B}_{R}}\left[q_{+}^{1 / 2}+|\nabla q|^{1 / 3}\right]$ and

$$
\mathrm{M}_{\Gamma}(u)=\sup _{\mathcal{B}_{R}}\left[\frac{u \Gamma^{\prime}(u)+\log (u / D)\left[\Gamma(u)-u \Gamma^{\prime}(u)\right]}{u[1-\log (u / D)]}\right]_{+} .
$$

Similar to the evolutionary case in Corollary 2.7, by passing to the limit $R \nearrow \infty$, we obtain a global gradient estimates for positive bounded solutions to Eq. 4.1, i.e., solutions $u$ satisfying the global bound $0<u \leq D$ on $M$ and subject to $\mathscr{R} i c_{f}^{m} \geq-\mathrm{k}_{m} g$ on $M$,

$$
\frac{|\nabla u|}{u} \leq C\left\{\sqrt{\mathrm{k}}+\mathrm{N}_{q}+\sqrt{\mathrm{M}_{\Gamma}(u)}\right\}\left(1-\log \frac{u}{D}\right) .
$$

A consequence of this global estimate is that if $\mathscr{R} i c_{f}^{m} \geq 0, q \equiv 0$ and $u$ is a positive bounded solution to $\mathscr{L}_{\Gamma}(u)=\Delta_{f} u+\Gamma(u)=0$ such that $\mathrm{M}_{\Gamma}=0$ then $u$ is a constant. In what follows we look at this more closely and examine some explicit examples of power-like and logarithmic type nonlinearities $\Gamma$ of particular interest in recent literature. The results will be stated under the curvature bound $\mathscr{R} i c_{f}(g) \geq 0$ (hence having $\mathrm{k}=0$ ) which is a weaker assumption than $\mathscr{R} i c_{f}^{m}(g) \geq 0$.

A quick comment on notation. Below we use p, q, r, s, etc. for real exponents, as, e.g., appearing in the specific choices of the nonlinearity $\Gamma$ (see below). We use $q=q(x, t)$ as before to denote the function in Eq. 1.1 with $\square_{q}=\partial_{t}-q-\Delta_{f}$ the $q$-weighted heat operator. A, B, C, etc. will denote arbitrary constants.

\subsection{The Choice $\Gamma(u)=A u \gamma(\log u)+B u^{p}+C u^{q}$ with real $p, q$}

Here we consider $\Gamma$ as described with three closely related choices $\gamma_{1}(s)=s^{\alpha}$ (integer $\alpha \geq 1), \gamma_{2}(s)=|s|^{\alpha}$ (real $\alpha>1$ ) and $\gamma_{3}(s)=|s|^{\alpha-1} s$ (real $\alpha>1$ ). The functions $\gamma(\log u)$ here all correspond to a logarithmic $\alpha$-power type nonlinearity but with different sign-changing structures. Also restricting to $\alpha>1$ in the second and third cases is to ensure continuous differentiability of $\Gamma$ and having the modulus sign in $|\log u|$ is prompted by taking positive real powers and the sign-changing nature of $\log u$ for $u>0$.

Let us proceed by first splitting $\Gamma$ by separating the logarithmic and the power terms and writing $\Gamma=\Gamma_{\gamma}+\Gamma_{\mathrm{pq}}$. For $\Gamma_{\gamma}=\mathrm{A} u \gamma(\log u)$ it is evident that $\Gamma_{\gamma}^{\prime}(u)=\mathrm{A}\left[\gamma+\gamma^{\prime}\right]$ and so with $s=\log u$

$$
\frac{u \Gamma_{\gamma}^{\prime}(u)+h\left[\Gamma_{\gamma}(u)-u \Gamma_{\gamma}^{\prime}(u)\right]}{(1-h) u}=\mathrm{A} \frac{\gamma+(1-h) \gamma^{\prime}}{1-h}=\mathrm{A} \frac{\left(\gamma-s \gamma^{\prime}\right)+\gamma^{\prime} \log (e D)}{1-h},
$$

where in writing the last identity we have made use of $h=\log (u / D)$. In a similar way for $\Gamma_{\mathrm{pq}}=\mathrm{B} u^{\mathrm{p}}+\mathrm{C} u^{\mathrm{q}}$ a straightforward calculation gives

$$
\frac{u \Gamma_{\mathrm{pq}}^{\prime}(u)+h\left[\Gamma_{\mathrm{pq}}(u)-u \Gamma_{\mathrm{pq}}^{\prime}(u)\right]}{(1-h) u}=\mathrm{B} \frac{\mathrm{p}(1-h)+h}{1-h} u^{\mathrm{p}-1}+\mathrm{C} \frac{\mathrm{q}(1-h)+h}{1-h} u^{\mathrm{q}-1} .
$$


Let $X_{\alpha}(u)=(1-\alpha) \log u+\alpha \log (e D)$. Then for $\alpha \geq 1,0<u \leq D$ we have $X_{\alpha}(u) \geq 0$. Now calculating explicitly the expression in Eq. 4.5 for each of the three cases of $\gamma$ it is seen that (with $\tilde{\gamma}=\gamma(s) / s$ )

$$
\mathrm{A} \frac{\left(\gamma-s \gamma^{\prime}\right)+\gamma^{\prime} \log (e D)}{1-h}=\mathrm{A} X_{\alpha}(u) \frac{\tilde{\gamma}(\log u)}{1-h} .
$$

Now putting the two segments $\Gamma_{\gamma}, \Gamma_{\mathrm{pq}}$ together we obtain

$$
\frac{u \Gamma^{\prime}(u)+h\left[\Gamma(u)-u \Gamma^{\prime}(u)\right]}{(1-h) u}=\mathrm{A} X_{\alpha} \frac{\tilde{\gamma}(\log u)}{1-h}+\mathrm{B} \frac{\mathrm{p}(1-h)+h}{1-h} u^{\mathrm{p}-1}+\mathrm{C} \frac{\mathrm{q}(1-h)+h}{1-h} u^{\mathrm{q}-1}
$$

and so by taking positive parts and using sub-additivity

$$
\begin{aligned}
& {\left[\frac{u \Gamma^{\prime}(u)+h\left[\Gamma(u)-u \Gamma^{\prime}(u)\right]}{(1-h) u}\right]_{+}} \\
& \quad=\left[\mathrm{A} X_{\alpha}(u) \frac{\tilde{\gamma}(\log u)}{1-h}+\mathrm{B} u^{\mathrm{p}-1}\left\{\mathrm{p}+\frac{h}{1-h}\right\}+\mathrm{C} u^{\mathrm{q}-1}\left\{\mathrm{q}+\frac{h}{1-h}\right\}\right]_{+} \\
& \leq[\mathrm{A} \tilde{\gamma}(\log u)]_{+} \frac{X_{\alpha}(u)}{1-h}+[\mathrm{B}(\mathrm{p}-h(\mathrm{p}-1))]_{+} \frac{u^{\mathrm{p}-1}}{1-h}+[\mathrm{C}(\mathrm{q}(1-h)+h)]_{+} \frac{u^{\mathrm{q}-1}}{1-h} .
\end{aligned}
$$

Based on this analysis and for the sake of the next theorem let us now formulate the following set of assumptions for non-zero $A$ in each of the three cases described above.

1. $\Gamma_{1}^{\gamma}(u)=\mathrm{A} u(\log u)^{\alpha}$ (integer $\left.\alpha \geq 1\right)$. Assume that either $\alpha$ is odd and $\mathrm{A}<0$ or $\alpha$ is even and either $\mathrm{A}<0$ and $1 \leq u \leq D$ or $\mathrm{A}>0$ and $0<u \leq 1$. Here in Eq. 4.8 $[\mathrm{A} \tilde{\gamma}(\log u)]_{+}=\left[\mathrm{A}(\log u)^{\alpha-1}\right]_{+}$and under these assumptions $[\mathrm{A} \tilde{\gamma}(\log u)]_{+}=0$.

2. $\Gamma_{2}^{\gamma}(u)=\mathrm{A} u|\log u|^{\alpha}$ (real $\left.\alpha>1\right)$. Assume that either $\mathrm{A}<0$ and $1 \leq u \leq D$ or $\mathrm{A}>0$ and $0<u \leq 1$. Here $[\mathrm{A} \tilde{\gamma}(\log u)]_{+}=\left[\mathrm{A}|\log u|^{\alpha-2} \log u\right]_{+}$and under these assumptions $[\mathrm{A} \tilde{\gamma}(\log u)]_{+}=0$.

3. $\Gamma_{3}^{\gamma}(u)=\mathrm{A} u|\log u|^{\alpha-1} \log u($ real $\alpha>1)$. Here $[\mathrm{A} \tilde{\gamma}(\log u)]_{+}=\left[\mathrm{A}|\log u|^{\alpha-1}\right]_{+}$and assuming $\mathrm{A}<0$ gives $[\mathrm{A} \tilde{\gamma}(\log u)]_{+}=0$.

Theorem 4.2 Let $\left(M, g, e^{-f} d v\right)$ be a weighted manifold with $\mathscr{R} i c_{f}(g) \geq 0$ and let $0<$ $u \leq D$ be a solution to the equation:

$$
\mathscr{L}_{\Gamma}(u)=\Delta_{f} u+\mathrm{A} u \gamma(\log u)+\mathrm{B} u^{\mathrm{p}}+\mathrm{C} u^{\mathrm{q}}=0,
$$

with $\gamma=\gamma_{1}, \gamma_{2}$ or $\gamma_{3}$ as introduced earlier. Assume A satisfies the conditions set above, $\mathrm{B} \leq 0, \mathrm{C} \geq 0, \mathrm{p} \geq 1$ and $\mathrm{q} \leq 0$. Then $u$ is a constant. Additionally if $\mathrm{A} \neq 0$ and $\mathrm{B}, \mathrm{C}=0$ then $u \equiv 1$.

Proof The assumptions on $\mathrm{A}$ and $u$ for each case of $\gamma$ give $[\mathrm{A} \tilde{\gamma}(\log u)]_{+}=0$ and those on $\mathrm{B}, \mathrm{C}, \mathrm{p}$ and $\mathrm{q}$ give $[\mathrm{B}(\mathrm{p}-h(\mathrm{p}-1))]_{+}=0$ and $[\mathrm{C}(\mathrm{q}(1-h)+h)]_{+}=0$. Referring to Eq. 4.8 this implies $M_{\Gamma}=0$ and from the global estimate Eq. 4.4 it follows that $\nabla u \equiv 0$. Hence $u$ is a constant. The last statement now follows by noting that the zero set of $\gamma$ is the singleton $\{s=0\}$. 


\subsection{The Choice $\Gamma(u)=\mathscr{P}(u)+\mathscr{R}(u)$}

Here we consider a power-like nonlinearity in $u$ where the functions $\mathscr{P}$ and $\mathscr{R}$ have the explicit form

$$
\mathscr{P}(u)=\sum_{j=1}^{N} a_{j} u^{\mathrm{p}_{j}}, \quad \mathscr{R}(u)=\sum_{j=1}^{N} b_{j} u^{\mathrm{q}_{j}}, \quad u>0,
$$

with real coefficients $a_{j}, b_{j}$ and exponents $\mathrm{p}_{j}, \mathrm{q}_{j}$ for $1 \leq j \leq N$. Now a direct calculation here gives

$$
\frac{(1-h) u \Gamma^{\prime}(u)+h \Gamma(u)}{(1-h)}=\sum_{j=1}^{N}\left\{a_{j} u^{\mathrm{p}_{j}}\left[\mathrm{p}_{j}+\frac{h}{1-h}\right]+b_{j} u^{\mathrm{q}_{j}}\left[\mathrm{q}_{j}+\frac{h}{1-h}\right]\right\}
$$

and so making note of the relation $h=\log (u / D)$ and taking positive parts gives

$$
\begin{aligned}
& {\left[\frac{(1-h) u \Gamma^{\prime}(u)+h \Gamma(u)}{(1-h) u}\right]_{+}=\left[\sum_{j=1}^{N}\left\{a_{j} \frac{\mathrm{p}_{j}-\left(\mathrm{p}_{j}-1\right) h}{1-h} u^{\mathrm{p}_{j}-1}+b_{j} \frac{\mathrm{q}_{j}(1-h)+h}{1-h} u^{\mathrm{q}_{j}-1}\right\}\right]_{+} } \\
\leq & \sum_{j=1}^{N}\left\{\left[a_{j}\left(\mathrm{p}_{j}-\left(\mathrm{p}_{j}-1\right) h\right)\right]_{+} \frac{u^{\mathrm{p}_{j}-1}}{1-h}+\left[b_{j}\left(\mathrm{q}_{j}(1-h)+h\right)\right]_{+} \frac{u^{\mathrm{q}_{j}-1}}{1-h}\right\} \\
\leq & \sum_{j=1}^{N}\left\{\left[a_{j}\left(\mathrm{p}_{j}-1\right) \log \left(\frac{D e^{\frac{\mathrm{p}_{j}}{\mathrm{p}_{j}-1}}}{u}\right)\right]_{+} \frac{u^{\mathrm{p}_{j}-1}}{1-h}+\left[b_{j}\left(\mathrm{q}_{j}-1\right) \log \left(\frac{D e^{\frac{\mathrm{q}_{j}}{\mathrm{q}_{j}-1}}}{u}\right)\right]_{+} \frac{u^{\mathrm{q}_{j}-1}}{1-h}\right\} .
\end{aligned}
$$

Now examining the last inequality it is seen that if $a_{j} \leq 0, b_{j} \geq 0$ and $\mathrm{p}_{j} \geq 1, \mathrm{q}_{j} \leq 0$, noting $0<u \leq D$, we have $\left[a_{j}\left(\mathrm{p}_{j}-\left(\mathrm{p}_{j}-1\right) h\right)\right]_{+}=\left[a_{j}\left(\mathrm{p}_{j}-1\right) \log \left(e^{\mathrm{p}_{j} /\left(\mathrm{p}_{j}-1\right)} D u^{-1}\right)\right]_{+}=0$ and $\left[b_{j}\left(\mathrm{q}_{j}(1-h)+h\right)\right]_{+}=\left[b_{j}\left(\mathrm{q}_{j}-1\right) \log \left(e^{\mathrm{q}_{j} /\left(\mathrm{q}_{j}-1\right)} D u^{-1}\right)\right]_{+}=0$. As a result each of the individual terms on the last line of Eq. 4.12 is zero and subsequently $\mathrm{M}_{\Gamma} \equiv 0$.

Theorem 4.3 Let $\left(M, g, e^{-f} d v\right)$ be a weighted manifold with $\mathscr{R} i c_{f}(g) \geq 0$ and suppose that $u$ is a bounded positive solution to the equation

$$
\mathscr{L}_{\Gamma}(u)=\Delta_{f} u+\mathscr{P}(u)+\mathscr{R}(u)=0 .
$$

with $\mathscr{P}, \mathscr{R}$ as in Eq. 4.10. Then if $a_{j} \leq 0, b_{j} \geq 0, \mathrm{p}_{j} \geq 1$ and $\mathrm{q}_{j} \leq 0$ then $u$ is a constant.

Proof From the discussion preceding the theorem, the assumptions on the exponents and coefficients of $\mathscr{P}$ and $\mathscr{R}$ give $\mathrm{M}_{\Gamma} \equiv 0$. Hence from the global form of the elliptic estimate Eq. 4.4 with $\mathrm{k}=0$ it follows that $\nabla u \equiv 0$ and hence the conclusion follows.

Remark 4.4 The conclusion of Theorem 4.3 admits the following nice refinement. We discuss this for $\Gamma=A u^{\mathrm{p}}$ as for $\Gamma=B u^{\mathrm{q}}$ the conclusion is similar and follows by putting the two parts together. Indeed here Eq. $4.12=\left[A(\mathrm{p}-1) \log \left(e^{\mathrm{p} /(\mathrm{p}-1)} D u^{-1}\right)\right]_{+} u^{\mathrm{p}-1} /(1-h)$. Now when $\mathrm{p} \geq 1$ or $\mathrm{p} \leq 0$, in view of $u \leq D$, we have $\log \left(e^{\mathrm{p} /(\mathrm{p}-1)} D u^{-1}\right) \geq 0$ and so as seen $\mathrm{M}_{\Gamma}=0$ iff $A$ and $\mathrm{p}-1$ have opposite signs. When $0 \leq \mathrm{p} \leq 1$, one can extend the conclusion to $A \leq 0$ if $e^{\mathrm{p} /(\mathrm{p}-1)} D \leq u \leq D$ which is an interesting observation in its own right. Here again $\mathrm{M}_{\Gamma}=0$. 


\subsection{The Choice $\Gamma(u)=A u^{s} \log u+B u^{p}+C u^{q}$ with real exponents s, $p$, $q$}

Here a straightforward differentiation gives $\Gamma^{\prime}(u)=\mathrm{A}\left(\mathrm{s} u^{\mathrm{s}-1} \log u+u^{\mathrm{s}-1}\right)+\mathrm{Bp} u^{\mathrm{p}-1}+$ $\mathrm{Cq} u^{\mathrm{q}-1}$ and so by substitution and arrangement of terms it follows that

$$
\begin{aligned}
& \frac{(1-h) u \Gamma^{\prime}(u)+h \Gamma(u)}{(1-h) u} \\
= & \mathrm{A} u^{\mathrm{s}-1}\left[\left(\mathrm{~s}+\frac{h}{1-h}\right) \log u+1\right]+\mathrm{B} u^{\mathrm{p}-1}\left[\mathrm{p}+\frac{h}{1-h}\right]+\mathrm{C} u^{\mathrm{q}-1}\left[\mathrm{q}+\frac{h}{1-h}\right] \\
= & \mathrm{A} u^{\mathrm{s}-1}\left[\frac{\mathrm{s}(1-h)+h}{1-h} \log u+1\right]+\mathrm{B} u^{\mathrm{p}-1}\left[\frac{\mathrm{p}(1-h)+\mathrm{p}}{1-h}\right]+\mathrm{C} u^{\mathrm{q}-1}\left[\frac{\mathrm{q}(1-h)+\mathrm{q}}{1-h}\right] .
\end{aligned}
$$

Let $X(\mathrm{~s})=[\mathrm{s}-h(\mathrm{~s}-1)] /(1-h)$. Then evidently $X(\mathrm{~s}) \geq 0$ when $\mathrm{s} \geq 1$ and $X(\mathrm{~s}) \leq 0$ when $\mathrm{s} \leq 0$. Furthermore Eq. $4.14=\mathrm{A}(X(\mathrm{~s}) \log u+1) u^{\mathrm{s}-1}+\mathrm{B} X(\mathrm{p}) u^{\overline{\mathrm{p}}-1}+\mathrm{C} X(\mathrm{q}) u^{\overline{\mathrm{q}}-1}$ and hence $[\text { Eq. } 4.14]_{+} \leq[\mathrm{A}(X(\mathrm{~s}) \log u+1)]_{+} u^{\mathrm{s}-1}+[\mathrm{B} X(\mathrm{p})]_{+} u^{\mathrm{p}-1}+[\mathrm{C} X(\mathrm{q})]_{+} u^{\mathrm{q}-1}$. Consider next the function

$$
\zeta_{\mathrm{s}}(h)=-\frac{1}{X(\mathrm{~s})}=\frac{1-h}{(\mathrm{~s}-1) h-\mathrm{s}}, \quad h \leq 0 .
$$

As for $0<\mathrm{s}<1$ the singularity of the functions $\zeta_{\mathrm{s}}$ at $h=\mathrm{s} /(\mathrm{s}-1)$ lies on the halfline $h<0$, hereafter, we restrict attention to $\mathrm{s}$ outside this interval, i.e., $\mathrm{s} \notin(0,1)$. Now it is easily seen that $\zeta_{\mathrm{s}}^{\prime}>0$ and so $\zeta_{\mathrm{s}}$ is monotonically increasing on $h \leq 0$. Moreover $\zeta_{\mathrm{s}}(0)=-1 / \mathrm{s}$ and $\lim \zeta_{\mathrm{s}}(h)=1 /(1-\mathrm{s})$ as $h \searrow-\infty$. Therefore when $\mathrm{s} \geq 1$ we have $1 /(1-\mathrm{s}) \leq \zeta_{\mathrm{s}}(h) \leq-1 / \mathrm{s}<0$ and when $\mathrm{s} \leq 0$ we have $0<1 /(1-\mathrm{s}) \leq \zeta_{\mathrm{s}}(h) \leq-1 / \mathrm{s}$. Now since when $\mathrm{A}$ and $X(\mathrm{~s}) \log u+1$ have opposite signs $[\mathrm{A}(X(\mathrm{~s}) \log u+1)]_{+}=0$ we can, based on the above argument, analyse this further and deduce the following:

- $\mathrm{A} \leq 0$ and $X(\mathrm{~s}) \log u+1 \geq 0$ :

1. If $\mathrm{s} \leq 0$ then $X(\mathrm{~s})<0$ and so $\log u \leq 1 /(1-\mathrm{s})$ gives $\log u \leq \zeta_{\mathrm{s}}=-1 / X(\mathrm{~s})$.

2. If $\mathrm{s} \geq 1$ then $X(\mathrm{~s})>0$ and so $\log u \geq-1 / \mathrm{s}$ gives $\log u \geq \zeta_{\mathrm{s}}=-1 / X$ (s).

- $\quad \mathrm{A} \geq 0$ and $X(\mathrm{~s}) \log u+1 \leq 0$ :

1. If $\mathrm{s} \leq 0$ then $X(\mathrm{~s})<0$ and so $\log u \geq-1 / \mathrm{s}$ gives $\log u \geq \zeta_{\mathrm{s}}=-1 / X(\mathrm{~s})$.

2. If $\mathrm{s} \geq 1$ then $X(\mathrm{~s})>0$ and so $\log u \leq 1 /(1-\mathrm{s})$ gives $\log u \leq \zeta_{\mathrm{s}}=-1 / X(\mathrm{~s})$.

Motivated by the above let us consider the following set of conditions on the coefficients $A, B, C$ and the exponents $s, p, q$ :

$$
\left\{\begin{array} { l } 
{ \mathrm { A } > 0 \text { and either: } } \\
{ \mathrm { s } \leq 0 \text { and } u \geq e ^ { - 1 / \mathrm { s } } , \text { or } } \\
{ \mathrm { s } \geq 1 \text { and } 0 < u \leq e ^ { 1 / ( 1 - \mathrm { s } ) } , }
\end{array} \quad \left\{\begin{array}{l}
\mathrm{A}<0 \text { and either: } \\
\mathrm{s} \geq 1 \text { and } u \geq e^{-1 / \mathrm{s}}, \text { or } \\
\mathrm{s} \leq 0 \text { and } 0<u \leq e^{1 /(1-\mathrm{s})}
\end{array}\right.\right.
$$

Theorem 4.5 Let $\left(M, g, e^{-f} d v\right)$ be a weighted manifold with $\mathscr{R} i c_{f}(g) \geq 0$. Assume that $u$ is a positive bounded solution to the equation (with real exponents $\mathrm{s}, \mathrm{p}, \mathrm{q}$ ):

$$
\mathscr{L}_{\Gamma}(u)=\Delta_{f} u+\mathrm{A} u^{\mathrm{s}} \log u+\mathrm{B} u^{\mathrm{p}}+\mathrm{C} u^{\mathrm{q}}=0,
$$

with $\mathrm{A}$ satisfying either set of assumptions in Eq. 4.15, B $\leq 0, \mathrm{C} \geq 0, \mathrm{p} \geq 1$ and $\mathrm{q} \leq 0$. Then $u$ is a constant. In particular when $\mathrm{A} \neq 0$ and $\mathrm{B}=0, \mathrm{C}=0$ then $u \equiv 1$. 
Proof Since under either set of conditions in Eq. 4.15 we have $[\mathrm{A}(X(\mathrm{~s}) \log u+1)]_{+}=0$ and likewise under the assumptions on $B, C$ and $p, q$ we have $[B X(p)]_{+}=[C X(q)]_{+}=0$ the assertion follows at once from the global gradient estimate as before.

\section{Further Global Bounds and Estimates}

Let $u$ be a positive solution to $\square u=\left(\partial_{t}-\Delta_{f}\right) u=\Gamma(u)$. For $\mathrm{p} \geq 2$ and $\mathrm{q} \in \mathbb{R}$ a pair of exponents and $\phi=\phi(t)$ a non-negative, smooth by otherwise arbitrary function set $\mathscr{R}_{\phi}^{\mathrm{p}, \mathrm{q}}[u]=\phi(t)|\nabla u|^{\mathrm{p}}+X u^{\mathrm{q}}$ where $X>0$ is a suitable constant.

Lemma 5.1 For $\mathrm{p}, \mathrm{q}, \phi, X$ as above $\mathscr{R}_{\phi}^{\mathrm{p}, \mathrm{q}}[u]$ satisfies the evolution identity

$$
\begin{aligned}
\square \mathscr{R}_{\phi}^{\mathrm{p}, \mathrm{q}}[u]= & \phi^{\prime}|\nabla u|^{\mathrm{p}}+\frac{\mathrm{p}}{2} \phi|\nabla u|^{\mathrm{p}-2}\left(-\left[\partial_{t} g+2 \mathscr{R} i c_{f}\right](\nabla u, \nabla u)+2\langle\nabla u, \nabla \Gamma(u)\rangle-2\left|\nabla^{2} u\right|^{2}\right) \\
& -\frac{\mathrm{p}}{2} \phi\left\langle\nabla|\nabla u|^{\mathrm{p}-2}, \nabla|\nabla u|^{2}\right\rangle+\mathrm{q} X\left[u \Gamma(u)-(\mathrm{q}-1)|\nabla u|^{2}\right] u^{\mathrm{q}-2} .
\end{aligned}
$$

In particular if the metric-potential pair evolve under the super Perelman-Ricci Eq. 1.14 then

$$
\square \mathscr{R}_{\phi}^{\mathrm{p}, \mathrm{q}}[u] \leq\left[\phi^{\prime}+\mathrm{p}\left(k+\Gamma^{\prime}\right) \phi\right]|\nabla u|^{\mathrm{p}}+\mathrm{q} X\left[u \Gamma-(\mathrm{q}-1)|\nabla u|^{2}\right] u^{\mathrm{q}-2} .
$$

Proof By a direct set of calculations it is straightforward to see that we have

$$
\left[\begin{array}{c}
\nabla \\
\partial_{t}
\end{array}\right] u^{\mathrm{q}}=\mathrm{q} u^{\mathrm{q}-1}\left[\begin{array}{c}
\nabla \\
\partial_{t}
\end{array}\right] u, \quad \Delta u^{\mathrm{q}}=\mathrm{q} u^{\mathrm{q}-1} \Delta u+\mathrm{q}(\mathrm{q}-1)|\nabla u|^{2} u^{\mathrm{q}-2}
$$

and so $\left(\partial_{t}-\Delta_{f}\right) u^{q}=q u^{q-1}\left(\partial_{t}-\Delta_{f}\right) u-q(q-1)|\nabla u|^{2} u^{q-2}$. Likewise for the gradient term

$$
\begin{aligned}
{\left[\begin{array}{c}
\nabla \\
\partial_{t}
\end{array}\right]|\nabla u|^{\mathrm{p}} } & =\frac{\mathrm{p}}{2}|\nabla u|^{\mathrm{p}-2}\left[\begin{array}{c}
\nabla \\
\partial_{t}
\end{array}\right]|\nabla u|^{2} \\
& =\frac{\mathrm{p}}{2}|\nabla u|^{\mathrm{p}-2}\left[\begin{array}{c}
\nabla|\nabla u|^{2} \\
-\partial_{t} g+2\left\langle\nabla u, \nabla \partial_{t} u\right\rangle
\end{array}\right] .
\end{aligned}
$$

where we have written $\partial_{t} g=\left[\partial_{t} g\right](\nabla u, \nabla u)$ for the sake of brevity. Now applying the divergence operator to the first row in Eq. 5.4 gives

$$
\Delta|\nabla u|^{\mathrm{p}}=\frac{\mathrm{p}}{2}|\nabla u|^{\mathrm{p}-2} \Delta|\nabla u|^{2}+\frac{\mathrm{p}}{2}\left\langle\nabla|\nabla u|^{\mathrm{p}-2}, \nabla|\nabla u|^{2}\right\rangle,
$$

and subsequently

$$
\Delta_{f}|\nabla u|^{\mathrm{p}}=\Delta|\nabla u|^{\mathrm{p}}-\left\langle\nabla f, \nabla|\nabla u|^{\mathrm{p}}\right\rangle=\frac{\mathrm{p}}{2}|\nabla u|^{\mathrm{p}-2} \Delta_{f}|\nabla u|^{2}+\frac{\mathrm{p}}{2}\left\langle\nabla|\nabla u|^{\mathrm{p}-2}, \nabla|\nabla u|^{2}\right\rangle .
$$

Thus using the above relation in conjunction with the second row in Eq. 5.4 gives

$$
\left(\partial_{t}-\Delta_{f}\right)|\nabla u|^{\mathrm{p}}=\frac{\mathrm{p}}{2}|\nabla u|^{\mathrm{p}-2}\left(-\partial_{t} g+2\left\langle\nabla u, \nabla \partial_{t} u\right\rangle-\Delta_{f}|\nabla u|^{2}\right)-\frac{\mathrm{p}}{2}\left\langle\nabla|\nabla u|^{\mathrm{p}-2}, \nabla|\nabla u|^{2}\right\rangle .
$$

Making note of Eq. 1.1 with $q(x, t)=0$ and the weighted Bochner-Weizenböck formula we have $2\left\langle\nabla u, \nabla \partial_{t} u\right\rangle-\Delta_{f}|\nabla u|^{2}=2\left\langle\nabla u, \nabla \Delta_{f} u+\nabla \Gamma\right\rangle-2 \mathscr{R} i c_{f}-2\left|\nabla^{2} u\right|^{2}-$ 
$2\left\langle\nabla u, \nabla \Delta_{f} u\right\rangle$ where again for brevity we have written $\mathscr{R} i c_{f}=\mathscr{R} i c_{f}(\nabla u, \nabla u)$. Hence after simplifying terms it follows that

$$
\begin{aligned}
\left(\partial_{t}-\Delta_{f}\right)|\nabla u|^{\mathrm{p}}= & \frac{\mathrm{p}}{2}|\nabla u|^{\mathrm{p}-2}\left(-\left[\partial_{t} g+2 \mathscr{R} i c_{f}\right]+2\langle\nabla u, \nabla \Gamma\rangle-2\left|\nabla^{2} u\right|^{2}\right) \\
& -\frac{\mathrm{p}}{2}\left\langle\nabla|\nabla u|^{\mathrm{p}-2}, \nabla|\nabla u|^{2}\right\rangle .
\end{aligned}
$$

Putting together Eq. 5.3 and Eq. 5.6 now gives

$$
\begin{aligned}
\square \mathscr{R}_{\phi}^{\mathrm{p}, \mathrm{q}}[u]= & \left(\partial_{t}-\Delta_{f}\right)\left[\phi|\nabla u|^{\mathrm{p}}+X u^{\mathrm{q}}\right] \\
= & \phi^{\prime}|\nabla u|^{\mathrm{p}}+\phi\left(\partial_{t}-\Delta_{f}\right)|\nabla u|^{\mathrm{p}}+X\left(\partial_{t}-\Delta_{f}\right) u^{\mathrm{q}} \\
= & \phi^{\prime}|\nabla u|^{\mathrm{p}}+\frac{\mathrm{p}}{2} \phi|\nabla u|^{\mathrm{p}-2}\left(-\left[\partial_{t} g+2 \mathscr{R} i c_{f}\right]+2\langle\nabla u, \nabla \Gamma(u)\rangle-2\left|\nabla^{2} u\right|^{2}\right) \\
& -\frac{\mathrm{p}}{2} \phi\left\langle\nabla|\nabla u|^{\mathrm{p}-2}, \nabla|\nabla u|^{2}\right\rangle+\mathrm{q} X\left[u \Gamma(u)-(\mathrm{q}-1)|\nabla u|^{2}\right] u^{\mathrm{q}-2}
\end{aligned}
$$

which is Eq. 5.1. To justify Eq. 5.2 it suffices to use the inequalities $\partial_{t} g+2 \mathscr{R i c} f \geq-2 \mathrm{~kg}$ (for any $m$ ), $\left|\nabla^{2} u\right|^{2} \geq 0$ and $\left\langle\nabla|\nabla u|^{\mathrm{p}-2}, \nabla|\nabla u|^{2}\right\rangle \geq 0$ when $\mathrm{p} \geq 2$. For this last inequality one just argues by writing $U=|\nabla u|^{2}$ and setting $\alpha=(\mathrm{p}-2) / 2$ and noting that the expression is $\left\langle\nabla U^{\alpha}, \nabla U\right\rangle=\alpha U^{\alpha-1}|\nabla U|^{2} \geq 0$.

We now give two consequences of this evolution identity to global gradient estimates. In both these statements $u$ is taken a positive solution to Eq. 1.1 with $q(x, t)=0$ and the metric-potential pair is assumed to evolve under a $(k, m)$ super Perelman-Ricci flow. Moreover $M$ is taken close.

Corollary 5.2 Under the assumptions of Lemma 5.1, if $q \geq 1$ and $\Gamma \leq 0, \Gamma^{\prime} \leq-a$ along $u$ then for $x \in M$ and $0<t \leq T$

$$
|\nabla u|(x, t) \leq e^{(k-a) t}\left\{\max _{M}\left[|\nabla u|^{\mathrm{p}}+u^{\mathrm{q}}\right](x, 0)-u^{\mathrm{q}}(x, t)\right\}^{1 / \mathrm{p}}
$$

Proof Starting from Eq. 5.2 and making note of $\Gamma \leq 0, \Gamma^{\prime} \leq-a$ for some $a$ we can write

$$
\begin{aligned}
\square \mathscr{R}_{\phi}^{\mathrm{p}, \mathrm{q}}[u] & \leq\left[\phi^{\prime}+\mathrm{p}(k-a) \phi\right]|\nabla u|^{\mathrm{p}}+\mathrm{q} X u^{\mathrm{q}-1} \Gamma(u)-\mathrm{q}(\mathrm{q}-1) X|\nabla u|^{2} u^{\mathrm{q}-2} \\
& \leq\left[\phi^{\prime}+\mathrm{p}(k-a) \phi\right]|\nabla u|^{\mathrm{p}}-\mathrm{q}(\mathrm{q}-1) X|\nabla u|^{2} u^{\mathrm{q}-2} .
\end{aligned}
$$

Now $\phi(t)=X e^{-\mathrm{p}(k-a) t}$ is non-negative, smooth and satisfies $\phi^{\prime}+\mathrm{p}(k-a) \phi=0$. Thus by substituting and noting $\mathrm{q} \geq 1$ it follow that $\square \mathscr{R}_{\phi}^{\mathrm{p}, \mathrm{q}} \leq 0$. The conclusion now follows by noting that due to the weak maximum principle $\mathscr{R}_{\phi}^{\mathrm{p}, \mathrm{q}}[u](x, t) \leq \sup \mathscr{R}_{\phi}^{\mathrm{p}, \mathrm{q}}[u](x, 0)$.

Corollary 5.3 Under the assumptions of Lemma 5.1 if $\Gamma, \Gamma^{\prime} \leq 0$ along $u$ and Eq. 1.14 holds with $k \geq 0$ then for $x \in M$ and $0<t \leq T$

$$
|\nabla u|^{2}(x, t) \leq \frac{1+2 k t}{2 t}\left[\max _{M} u^{2}(x, 0)-u^{2}(x, t)\right] .
$$


Proof Setting $\mathrm{p}=2, \mathrm{q}=2$ and $X=1 / 2$ in Eq. 5.2 gives

$$
\begin{aligned}
\square \mathscr{R}_{\phi}^{2,2} & =\square\left[\phi|\nabla u|^{2}+X u^{2}\right] \\
& \leq\left[\phi^{\prime}+2\left(k+\Gamma^{\prime}\right) \phi\right]|\nabla u|^{2}+2 X\left[u \Gamma-|\nabla u|^{2}\right] \\
& \leq\left(\phi^{\prime}+2 k \phi-1\right)|\nabla u|^{2}+2 \Gamma^{\prime} \phi|\nabla u|^{2}+u \Gamma .
\end{aligned}
$$

Now when $k \geq 0$ by taking $\phi(t)=t /(1+2 k t)$ we have $\left(\phi^{\prime}+2 k \phi-1\right) \leq 0$ and so subject to $\Gamma, \Gamma^{\prime} \leq 0$ we have $\square \mathscr{R}_{\phi}^{2,2} \leq 0$. The conclusion now follows by an application of the weak maximum principle.

Acknowledgements The author wishes to thank the anonymous referee for a careful reading of the manuscript and useful comments.

Data Availability Statement The author declares that all the data supporting the findings of this study are available within the article.

Open Access This article is licensed under a Creative Commons Attribution 4.0 International License, which permits use, sharing, adaptation, distribution and reproduction in any medium or format, as long as you give appropriate credit to the original author(s) and the source, provide a link to the Creative Commons licence, and indicate if changes were made. The images or other third party material in this article are included in the article's Creative Commons licence, unless indicated otherwise in a credit line to the material. If material is not included in the article's Creative Commons licence and your intended use is not permitted by statutory regulation or exceeds the permitted use, you will need to obtain permission directly from the copyright holder. To view a copy of this licence, visit http://creativecommons.org/licenses/by/4.0/.

\section{References}

1. Abolarinwa, A., Taheri, A.: Elliptic gradient estimates for a nonlinear $f$-heat equation on weighted manifolds with evolving metrics and potentials. Chaos Solitons Fractals 142(1), Special issue: Sing. Evol. Eq., Ed: M. Ruzhansky (2021)

2. Abolarinwa, A., Taheri, A.: Geometric estimates on weighted $p$-fundamental tone and applications to the first eigenvalue of submanifolds with bounded mean curvature, Complex Var. Elliptic Equ., Published online (2021)

3. Băileşteanu, M., Cao, X., Pulemotov, A.: Gradient estimates for the heat equation under the Ricci flow. J. Funct. Anal. 258, 3517-3542 (2010)

4. Bakry, D.: L'hypercontractivité Et Son Utilisation En Théorie Des Semigroupes. In: Lecture Notes in Math, vol. 1581, pp. 1-114. Springer, Berlin (1994)

5. Bakry, D., Émery, M.: Diffusions Hypercontractives. In: Azḿa, J., Yor, M. (eds.) Séminaire De ProbabilitéS XIX 1983/84. Lecture Notes in Mathematics. Springer, Berlin (1123)

6. Bakry, D., Gentil, I., Ledoux, M.: Analysis and geometry of Markov diffusion operators, a series of comprehensive studies in mathematics, vol. 348. Springer, Berlin (2012)

7. Biduat-Vèron, M.F., Veròn, L.: Nonlinear elliptic equations on compact Riemannian manifolds and asymptotics of the Emden equations. Invent. Math. 106, 489-539 (1991)

8. Brighton, K.: A Liouville theorem for smooth metric measure spaces. J. Geom. Anal. 23, 562-570 (2013)

9. Choquet-Bruhat, Y.: General Relativity and the Einstein Equations. OUP, Oxford Mathematical Monographs (2009)

10. Chow, B., Lu, P., Nei, L.: Hamilton's Ricci flow, graduate studies in mathematics, vol. 77. AMS (2007)

11. Gilbarg, D., Trudinger, N. Elliptic Partial Differential Equations of Second Order. Classics in Mathematics, 2nd edn. Springer, Berlin (2013)

12. Grigor'yan, A.: Heat kernel analysis on manifolds. Studies in Advanced Mathematics AMS (2013)

13. Dung, N.T., Khanh, N.N., Ngô, Q.A.: Gradient estimates for $f$-heat equations driven by Lichnerowicz's equation on complete smooth metric measure spaces. Manuscripta Math. 155, 471-501 (2018)

14. Hamilton, R.: The formation of singularities in the Ricci flow. Surv. Diff. Geom. 2, 7-136 (1995)

15. Hamilton, R.: A matrix Harnack estimate for heat equation. Comm. Anal. Geom. 113-126 (1993)

16. Hamilton, R.: Three-Manifolds With positive Ricci curvature. J. Diff. Geom. 17, 255-306 (1982) 
17. Kotschwar, B.: Hamilton's gradient estimate for the heat kernel on complete manifolds. Proc. Amer. Math. Soc. 135, 3013-3019 (2007)

18. Li, P.: Geometric Analysis, Cambridge studies in advanced mathematics, 134 CUP (2012)

19. Li, P., Yau, S.T.: On the parabolic kernel of Schrödinger operator. Acta Math. 156, 153-201 (1986)

20. Li, S., Li, X.D.: $W$-entropy, super Perelman Ricci flows and $(K, m)$-Ricci solitons. J. Geom. Anal. 30, 3149-3180 (2020)

21. Li, S., Li, X.D.: Hamilton's differential Harnack inequality and the $\mathcal{W}$-entropy formula on complete Riemannian manifolds. J. Funct. Anal. 274, 3263-3290 (2018)

22. Li, S., Li, X.D.: On Harnack inequalities for Witten Laplacian on Riemannian manifolds with super Ricci flows, Asian. J. Math. 22, 577-598 (2018)

23. Li, X.D.: Liouville theorems for symmetric diffusion operators on complete Riemannian manifolds. J. Math. Pures Appl. 84, 1295-1361 (2005)

24. Lott, J.: Some geometric properties of the Bakry-Émery Ricci tensor. Comment. Math. Helv. 78, 865883 (2003)

25. Morrison, G., Taheri, A.: An infinite scale of incompressible twisting solutions to the nonlinear elliptic system $\mathscr{L}[; \mathrm{a}, \mathrm{B}]=\nabla \mathscr{P}$ and discriminant $\Delta(h, g)$. Nonlin. Anal. 173, 209-219 (2018)

26. Morrison, G., Taheri, A.: The interplay between two Euler-Lagrange operators relating to the nonlinear elliptic system $\Sigma[(u, \mathscr{P}), \Omega]$. Adv. Op. Th. 6, 1-28 (2021)

27. Müller, R.: Differential Harnack Inequalities and the Ricci Flow. EMS Series of Lectures in Mathematics, EMS (2006)

28. Perelman, G.: The entropy formula for the Ricci Flow and its geometric application, arxiv: math DG/0211159v1 (2002)

29. Saloff-Coste, L.: Aspects of Sobolev-Type inequalities. London Mathematical Society Lecture Note Series, 289 CUP (1984)

30. Schoen, R., Yau, S.T.: Lectures on Differential Geometry. International Press, Cambridge (1994)

31. Souplet, P., Zhang, Q.S.: Sharp gradient estimate, and Yau's Liouville theorem for the heat equation on noncompact manifolds. Bull. Lond. Math Soc. 38, 1045-1053 (2006)

32. Sturm, K.T.: Super-Ricci flows for metric measure spaces. J. Funct. Anal. 275, 3504-3569 (2018)

33. Taheri, A.: Function Spaces and Partial Differential Equations. Vol. I, Oxford Lecture Series in Mathematics and its Applications, 40 OUP (2015)

34. Taheri, A.: Function Spaces and Partial Differential Equations. Vol. II, Oxford Lecture Series in Mathematics and its Applications, 41 OUP (2015)

35. Taheri, A.: Liouville theorems and elliptic gradient estimates for a nonlinear parabolic equation involving the Witten Laplacian, Adv. Calc. Var., Published online (2021)

36. Villani, C.: Optimal Transport: Old and New, A series of comprehensive studies in mathematics, vol. 338. Springer, Berlin (2008)

37. Wang, W.: Harnack, inequality, heat kernel bounds and bounds, eigenvalue estimate under integral Ricci curvature. J. Diff. Eq. 269, 1243-1277 (2020)

38. Wei, G., Wylie, W.: Comparison geometry for the Bakry-Émery Ricci tensor. J. Diff. Geom. 83, 377-405 (2009)

39. Wu, J.Y.: Elliptic gradient estimates for a weighted heat equation and applications. Math. Z. 280, 451468 (2015)

40. Wu, J.Y:: Gradient estimates for a nonlinear parabolic equation and Liouville theorems. Manuscript Math. 159, 511-547 (2018)

41. Zhang, Q.S.: Some gradient estimates for the heat equation on domains and for an equation by Perelman. Int. Math. Res. Notices (2006)

42. Zhang, Q.S.: Sobolev inequalities, heat kernels under Ricci flow and the Poincaré conjecture CRC Press (2011)

Publisher's Note Springer Nature remains neutral with regard to jurisdictional claims in published maps and institutional affiliations. 